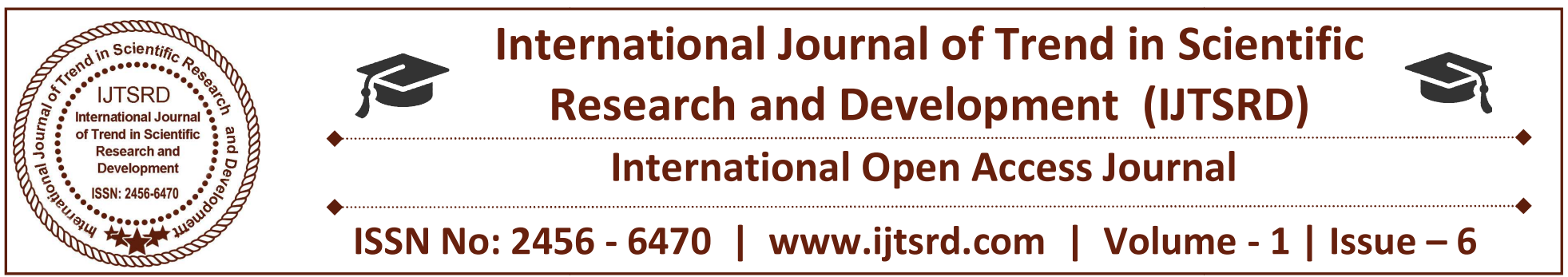

\title{
Minimal access oesophagectomy: Systematic review and sub-group meta-analyses based on Randomised Controlled trials
}

\author{
Dr. Syed SumairaNawaz \\ Consultant Surgeon \\ MBBS - GMC, Srinagar \\ MCh - Minimally invasive \& Robotic Surgery, United Kingdom
}

\begin{abstract}
Background: All studies conducted to compare minimal access oesophagectomy (MAO) with open oesophagectomy (OO) till date, have the limitation that they are primarily bas ed on non randomized studies. Only evidence based on randomised controlled trials (RC Ts) can establish MAO as the preferred surgery for resectable oesophageal cancer (OCA) and hence the current review.

Objectives: This review aims to establish a firm body of evidence in support of MAO. The objectives involve using PICO strategy and searching for relevant RCTs, extracting and analyzing data from them in order to derive conclusions that help establish evidence in favour of MAO.
\end{abstract}

Data Sources: of the current review are RCTs that asess outcomes of MAO.

Review Methodology: Quantitave study has been designed through a systematic review and metaanalyses of RCTs.

Results: Blood loss during surgery, post -operative pulmonary infection and duration of hospital stay favour MAO versus $\mathrm{OO}$ for resectable OCA management.

Conclusion:The ideal MAO strategy may involve thoracoscopic oesophagectomy in prone position with low tidal volume ventilation, perioperative administration of amino-acids and neutrophil elastase inhibitor plus immediate postoperative chest physiotherapy and enteral feeding.
Keywords: Minimal access oesophagectomy, Minimally invasive esophagectomy, Thoracoscopic oesophagectomy.

\section{INTRODUCTION}

Oesophageal cancer (OCA) is the cancer of the food pipe/gullet. It is the eighth common est tumour (Cancer Research UK, 2016) (Appendix 1) contribution being 4.9\% (Cancer today IARC, 2012). The incidence of oesophageal adenocarcinomas has quadrupled from 1960s to 1990s (Appendix 2) but the 5 year survival still remains less than 14\% (Enzinger and Mayer, 2003). Only one-third of OCA patients at the time of diagnosis have resectable tumour (AJCC TNM stage cT1-3 N0-1 M0) that is suitable for surgery (Appendix 3). 30\% of these also end up with microscopically residual disease. Curative surgery involves resecting part or whole of the oesophagus (Oesophagectomy) with restoration of continuity of the gastrointestinal tract subsequently (Park et al., 20 09). Traditionally open technique has been used to perform oesophagectomy. It causes substantial morbidity plus mortality. Increasing incidence of OCA and poor surgical out comes using open oesophagectomy (OO) lead to attempts to use minimal access surgical techniques instead (Smithers et al., 200 7). Minimal access surgery was found to be feasi ble for resectable OCA (Yamamoto et al., 2013). But some reluctance to use MAS instead of OO has continued till date for fear of inadequate oncological surgical resection of the advanced OCA (Titcomb et al., 2016). Currently only $16 \%$ oesophagectomies in $\mathrm{UK}$ are performed using MAS, the rest are open 
surgeries (Burdall et al., 2016). The main reason is that level 1 evidence in favour of MAS versus OO has not been provided till date. This can be obtained only through RCT based systematic review showing outcomes in favour of minimal access oesophagectomy (MAO) versus OO outcomes (Yamamo to et al., 2013).

\section{BACKGROUND}

\section{What is known (including any reviews) about the topic so far?}

Open oesophgectomy (OO) was the only known curative surgery for OCA till 1980s when laparoscopy and thoracoscopy were introduced. Cushieri et al. performed and described the first MAO in 1992 (Cuschieri, Shimi and Banting, 1992). More than 2 decades later, MAO is still not as popular as other MAS procedures. It continues to be regarded as the most complex gastrointestinal surgery. Surgeons reluctant to use MAO in place of OO debate about mortality, morbidity, oncological radicality as well as the cost involved (Nagpal et al., 2010). Clear proof of superiority of MAO over OO is not forthcoming since comparative studies till date are mostly unmatched patient cohorts. The evidence that has accumulated over the years began with Collard et al. in 1993 who attempted subtotal oesophagectomy by thoracoscopy and showed that thoracoscopic oesophageal resections can be as extensive as open (Broussard et al, 2016). Luketich et al in 2003 reported that MAO is associated with lower mortality and shorter hospital stay than OO (Kim et al., 2012). Rajan et al. reported their results after performing MAO in 463 patients from 1997 to 2009. Their statistics included operative mortality and overall morbidity. They used various MAO techniques and concluded that MAO is safe and the type of MAO needs to be tailored depending on the OCA level, stage and histology (Senthilna than et al., 2010). Nguyen et al reported their results after 104 MAOs from 1998 and 2007 that included thoracoscopic as well as laparoscopic MAOs. They concluded that MAO is feasible, has acceptable morbidity, is associated with lower conversion rates and lower mortality as compared to OO (Nguyen et al., 2008). Blood loss ,duration of in-hospital stay and ICU stay, overall complications as well as pulmonary complication rates are less in patients having MAO vs OO (Verghese et al., 2009). The limitations of this study were heterogeneity with regards to MAO techniques, selection bias and publication bias. Biere et al. conducted a meta-anal ysis that included one controlled clinical trial and 9 case-con trol studies (Biere, Cuesta and van der Peet, 2009). Nagpal et al., conducted a meta-analy sis comparing open and minimal access oesophagectomies and the results were same as those found by Verhages et al(Nagpal et al., 2010). The results of studies comparing MAO and OO were summarised in a table (Appendix 4) by Kimet al in 2012 in their study. All the above authors reached the same conclusion from their studies that MAO is feasi bile and safe surgical option for OCA management and comparable to OO. But the qual ity of their studies was poor. These studies are not best quality evidence to establish sup eriority of MAO over OO because they are predominantly based on case series which are regarded as low level evidence in the medical field. The authors have not compared open and minimal access oesophagectomies adequately. The studies are heterogenous with reg ard to the types of MAOs used hence lack generalizability. The studies have used various combinations of MAS and open techniques all of which contribute to heterogeneity. There is selection bias also as the patients selected for MAS were not representative of the general OCA patients. Publication bias may also be there. The limitation of all the systematic reviews and metaanalyses conducted to compare MAO with OO is that they are primarily based on non randomized studies. These meta- analyses came to the same conclusion that that prospective randomized controlled trials (RCTs) comparing MAO and $\mathrm{OO}$ are needed. Only evidence from a systematic review based on RCTs can establish MAO as the preferred surgical option for resectable OCA management.

What this review may add? The current review aims to gather high quality evidence in favour of MAO by analyzing all available RCTs that assess factors affecting the outcomes of MAO. The purpose of the review is not only to collect evidence that supports MAO but also to determine the best MAO strategy for OCA resection as MAO can be performed in conjuction with differing perioperative set-ups and by using combination of different techniques. Choosing the best combination in a given scenario is important to get opti mal surgical outcomes. This review aims to find the MAO strategy that will provide best possible surgical outcomes for resectable OCAs. So, all RCTs in which factors affecting any outcome of MAO are discussed have been included. MAO may involve 
thoracoscopy or laparoscopy or both or even robotic surgery. The patient positions in which MAO can be performed also vary and the results of MAO get affected by administration of different perioperative substances. Whether the patient receives neoadjuvant/adjuvant treatment, whether postoperative chest physiotherapy and enteral nutrition are given or not plus what tidal volume is given by the anaesthetist during MAO, all can affect the outcomes of MAO. Hence the RCTs involving these topics have been included in this review. This revi ew is important since all the reviews and meta analyses till date have been based on non -randomised studies which makes current evidence in favour of MAO of low quality.

\section{AIMS \& OBJECTIVES:}

The current review aims to establish a firm body of evidence in support of MAO for rese ctable OCA management. The review is based on the hypothesis that MAO is associated with statistically significant lower morbidity than OO. The optimal surgical technique for OCA needs to be determined by assessing all the factors which play a role in the MAO out comes. MAO can be advocated as the gold standard in management of resectable OCA by providing level 1 evidence based on RCTs. This can lead to improvement in the outco mes of OCA surgery which has been notorious for high morbidity. The objectives of the current study were planned using PICO framework:

$\mathrm{P}=$ Population $=$ Oesophageal cancer patients

$\mathrm{I}=$ Minimal access oesophagectomy/perioperative modifying factors

$\mathrm{C}=$ Control=Open oesophagectomy/absence of perioperative modifying factors

$\mathrm{O}=\mathrm{MAO}$ outcomes including primary and secondary outcome measures.

\section{The objectives are:}

search for relevant RCTs as per the search strategy in the review protocol, -extraction of relevant information from the selected trials about outcomes/factors affecting outcomes of MAO,

$>$ analysis of the information thus obtained and

$>$ discussion of the results in order to establish all available current evidence regarding the outcomes of MAO which may used to develop the ideal MAO protocol for resectable OCA management.

The study was conducted keeping in mind the PRISMA statement [APPENDIX 5].

\section{Methodology \& Protocol}

STUDY DESIGN -Basis of choosing the design of the current study \& justification of met hodology used: In 2009, a survey involving surgeons in 41 countries was carried out to determine the preferred surgical option for OCA management. It was found that $52 \%$ of responders preferred open thoracotomy, 26\% preferred transhiatal oesophagectomy and just $14 \%$ chose MAO. This proved that MAO was still very far from being adopted as surg ery of choice in OCA management (Boone et al., 2009). Authors of case series, case contr olled studies, cohort studies, systematic reviews and meta-analyses comparing MAO with OO have accepted in the conclusions that because of non-availability of high quality evi dence through prospective RCTs, superiority of MAO versus OO cannot be established (Dantoc, Cox and Eslick, 2012b). Level 1 evidence from systematic review of randomised controlled trials is considered the gold standard of medical knowledge (Hau ghom and Advisor, 2015). This is what has been lacking so far. To prefer MAO in resect able OCA management it is essential to generate level 1 evidence through a systematic review of RCTs that report in favour of MAO versus OO. Hence the current study design is systematic review of the relevant RCTs. All the selected RCTs were found to involve qu antitative data. The information from these trials needs to be analyzed and results obtain ed need to be combined in order to determine the best MAO strategy for managing resec table OCA. So, this systematic review concludes with a meta- analyses that aim to pool the results of the selected RCTs. Study Design is a quantitative. The systematic review has a positivistic paradigm .

\section{SEARCH STRATEGY}

Database search- A comprehensive search was carried out as is needed for a systema tic review. Electronic databases searched included Biomed Central, CINAHL plus, Coch rane library, Proquest, Pubmed, Science direct, Scopus, Web of science and 
Wiley. Other online sources searched included Journals (OVID journals, NHS journals library), Clini cal trial registers PROSPERO \& TRIP database. The internet search results can be seen in table 1 below.

Search terms- The review needed all trials on outcomes of minimal access oesophagec tomies. So all possible synonyms of the term "minimal access oesophagectomy" were used as key words/search terms. These included :

Minimal access oesophagectomy (Mao), Minimal access esophagectomy (Mae), Minimally invasive oesophagectomy (Mi0), Minimally invasive esophagectomy (Mie), Robotic oesophagectomy (R0), Robotic esophagectomy (Re), Robot-associated oesopha gectomy (Rao), Robot-associated esophagectomy (Rae), Laparoscopic oesophagectomy (Lo), Laparoscopic esophagectomy (Le), Thoracoscopic oesophagectomy (Tho), Thora coscopic esophagectomy (The) Table 1 below has these search terms in the headings of the columns.
Search restrictions-Organisations and topic experts could also not be contacted due to time limitation for the current study. Grey literature was also not searched for the same reason. Exclusion of nonEnglish, unpublished \& gray literature induces language bias, selection bias, publication bias \& location bias. Limited randomised controlled tri als on robot-assisted oesophagectomies made it impossible to include the outcomes of robotic oesophagectomies in the current study.

Journal Hand search involved looking in the university library for latest reports from health bodies, abstracts of conference presentations \& reviews. But hand search was too time consuming and was abandoned.

Organisations and topic experts were not contacted due to time limitation.

Bibliography search: The reference lists of the trials selected were checked for any other relevant study.

Table 1 below- shows search results on 1 may 2016 using the keywords in the corresponding databases.

\begin{tabular}{|c|c|c|c|c|c|c|c|c|c|c|c|c|}
\hline Keyword \& Database & Mao & Mae & Mi o & Mie & Lo & Le & Ro & $\operatorname{Re}$ & Rae & Rao & Tho & The \\
\hline Biomed Central & 27 & 63 & 12 & 4 & 13 & 45 & 4 & 14 & 10 & 1 & 3 & 26 \\
\hline Google scholar & 768 & 641 & 9420 & 8680 & 3280 & 13800 & 660 & 2350 & 2050 & 2190 & 6660 & 6930 \\
\hline MEDLINE & 1 & 3 & 70 & 431 & 27 & 187 & 4 & 34 & 26 & 4 & 34 & 273 \\
\hline CINAHL & 5 & 12 & 23 & 80 & 2 & 28 & 1 & 10 & 6 & 4 & 1 & 46 \\
\hline Cochrane Library & 1 & 1 & 12 & 18 & 1 & 2 & 0 & 0 & 1 & 0 & 6 & 24 \\
\hline OVID Journals & 805 & 805 & 1518 & 1518 & 1122 & 1122 & 332 & 332 & 388 & 388 & 263 & 263 \\
\hline Proquest central & 235 & 796 & 602 & 1997 & 526 & 1978 & 121 & 400 & 282 & 105 & 346 & 972 \\
\hline Pubmed & 23 & 23 & 819 & 819 & 680 & 680 & 105 & 105 & 50 & 50 & 481 & 481 \\
\hline Science direct & 103 & 631 & 248 & 2030 & 297 & 2223 & 61 & 469 & 189 & 21 & 107 & 1023 \\
\hline Scopus & 11 & 23 & 128 & 953 & 78 & 661 & 14 & 124 & 61 & 12 & 60 & 593 \\
\hline TRIP datab & 0 & 0 & 1 & 17 & 8 & 13 & 0 & 3 & 3 & 0 & 26 & 135 \\
\hline Web of science & 8 & 20 & 145 & 1094 & 70 & 627 & 12 & 121 & 58 & 11 & & 216 \\
\hline
\end{tabular}


Internet search strategy : The internet search strategy involved a scoping search first. During the scoping search, the term "minimal access oesophagectomy" was used in Goo le scholar so as to get an idea of what kind of literature is there online. Google scholar is an easily accessible and freely available database and hence the choice. This search prod uced more than 9000 results. It could be seen that there are articles, reviews and book chapters about this topic. Going through the abstracts of some articles revealed some co mmon conclusions that oesophageal cancer is a dreaded cancer for which the optimal sur gical management is still not known and there is requirement for RCT based evidence to establish the right place of MAO. Thus,the review seemed feasible and was planned to include trials only.
The initial search for this review using the keywords produced unmanagable numbers as can be seen in the table 1. Therefore the search needed to be refined.

Refined Search- The following search limits were applied:

Search in title/abstract/keyword,

$>$ Articles with abstracts,

$>$ In English language,

$>$ In humans,

$>$ In the field of Health sciences,

> Published during the years 2000-2016,

$>$ trials only

$>$ Boolean operator OR,AND were used with the keywords for refined search.

$>$ Refined search did not include Google scholar as it was impossible to apply all search limits

\begin{tabular}{|c|c|c|}
\hline Refined search in following DATABASEs & T & N \\
\hline [accessed through university library links in may 2016] & & 3 \\
\hline [MEDLINE \& CINAHL] & 164 & 1 \\
\hline SCIENCE Citation Index- search via Web of Science & 237 & 0 \\
\hline OVID journals (via EBSCO Host) & 109 & 1 \\
\hline PROQUEST CENTRAL(4 relevant databases searched) & 26 & 18 \\
\hline SCOPUS & 181 & 8 \\
\hline TRIP Database & 21 & 20 \\
\hline Cochrane central register of controlled trials & 32 & 57 \\
\hline EMBASE via PUBMED & 796 & \\
\hline Refined search results [in May 2016] & & \\
\hline
\end{tabular}

Table 2

$\mathrm{T}=$ Total number of articles produced by the refined search in the database.

$\mathrm{N}=$ No. after selecting Relevant articles within each database \& after removing duplicates in the particular database searched.

APPENDIX 8 at the end shows Completed search summary for EMBASE database full search to give an example of how serch was done.
Internet search strategy :(Prisma flow diagram below)

Outcome of the search process - Refined search provided total 796 articles as seen in table 2. Selection of relevant articles in each database and removing duplicates within databases brought this figure down to 57 articles. The 57 trials still included duplicates as the same articles were found in different databases. Removing duplicates and selecting trials that fitted the inclusion criteria lead to final selection of 12 RCTs for the current systematic review. 


\section{PRISMA flow diagram of refined search in the current study}

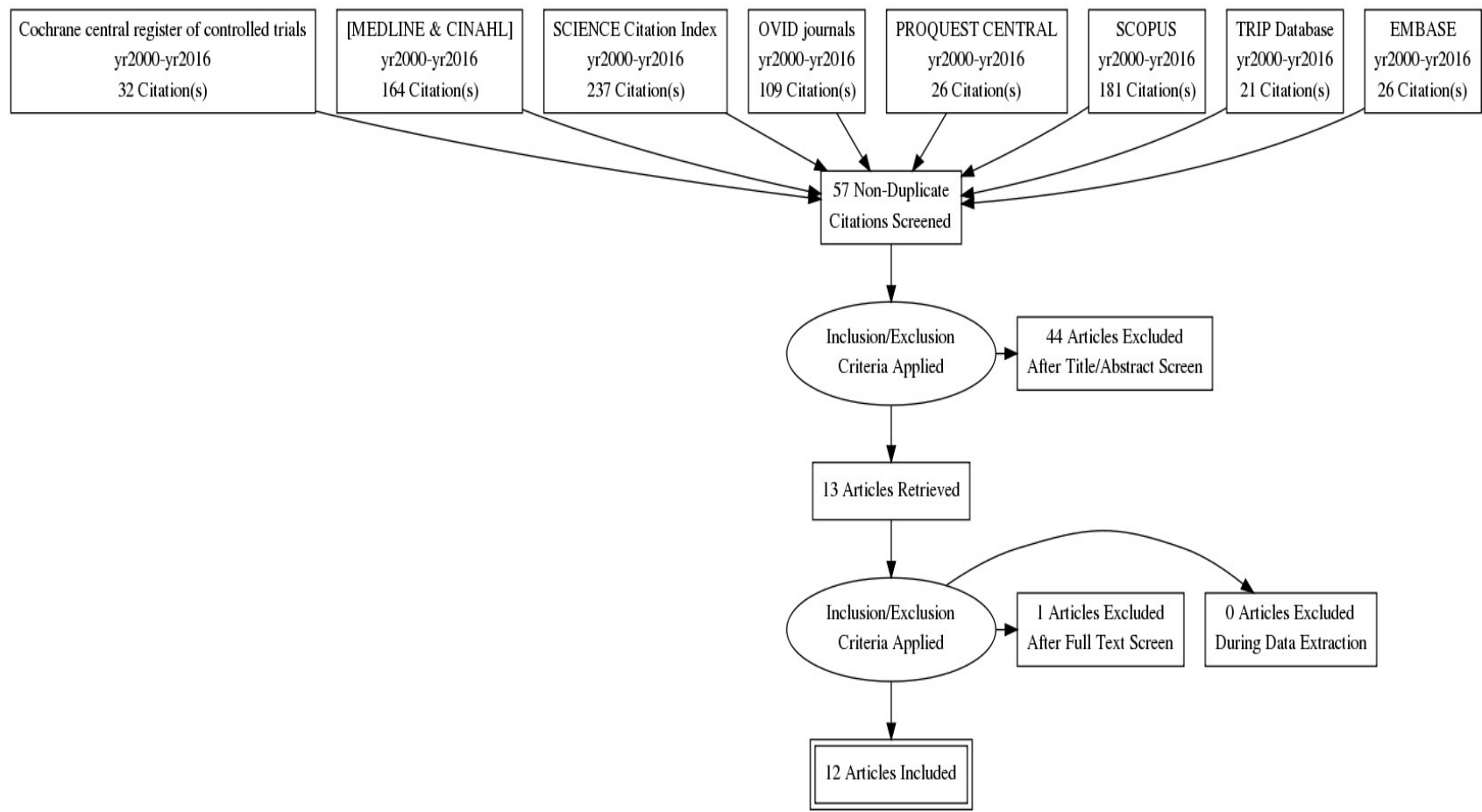

These twelve selected RCTs (Bibliography/ Reference list) are in Table 3 below.

Justification of the search strategy : Systematic reviews should aim to locate all studies that are relevant to the study questions. It is recommended that bibliographic searches for health care studies include databases MEDLINE and the Cochrane Central Register of Controlled Trials. Other data bases useful to search are EMBASE, CINAHL and Psych INFO (Relevo, 2012). Therefore, to locate all relevant studies for the current review, a comprehensive internet search of all the databases was carried out. Time period available to complete the review was limited and thus it was neither possible to contact experts nor to look for gray literature. All possible synonyms of the term "minimal access oesophagectomy" were used as key words /search terms because the review needed trials focussed on the outcomes of minimal access oesophagectomies. Although comparison with open oesophagectomy outcomes is involved in the meta-analysis part of the current review, the term "open oesophagectomy" or synonyms are not used as search terms. This is because comparison with open oesophagectomy was not the primary aim of the curren $t$ review. The review aimed to study the outcomes and factors affecting the outcomes of MAO by analysis of trials done so far. The comparison with $\mathrm{OO}$ was obvious in five trials and thus it became possible to do a meta-analysis involving these studies. However, the rest of the trials involve comparison of different factors affecting outcomes of MAO only; this heterogeneity lead to their exclusion from meta-analysis but inclusion in the systema tic review as all of them provide valid information in relation to outcomes of MAO. The trials about MAO were published only after the year 2000 when minimal access surgery started getting popular and therefore the search limit year 2000 to current. Current me ans May 2016 for this review search. Trials only were searched as the review aims to generate high quality evidence only by studying all relevant RCTs till date 
International Journal of Trend in Scientific Research and Development (IJTSRD) ISSN: 2456-6470

Table 3

\begin{tabular}{|c|c|c|}
\hline No. & Authors & Randomised controlled trial [ \& what the trial assessed ] \\
\hline 1. & Ito et al. & $\begin{array}{c}\text { Thoracoscopic oesophagectomy with chest physical therapy vs open } \\
\text { oesophagectomy with chest physical therapy [Effects on postoperative respiratory } \\
\text { complications were studied] }\end{array}$ \\
\hline 2. & $\begin{array}{l}\text { Ninom iya } \\
\text { et al }\end{array}$ & $\begin{array}{l}\text { Thoracoscopic esophagectomy with perioperative neutrophil elastase inhibitor } \\
\text { versus thoracoscopic esophagectomy without perioperative neutrophil elastase } \\
\text { inhibitor [Effects on postoperative complications were assessed.] }\end{array}$ \\
\hline 3. & Biere et al & $\begin{array}{c}\text { Minimal access oesophagectomy versus open procedure } \\
\text { [ Postoperative Outcomes were compared] }\end{array}$ \\
\hline 4. & $\begin{array}{l}\text { Wajed et } \\
\quad \text { al }\end{array}$ & $\begin{array}{l}\text { Minimal access oesophagectomy with prior Gastric ischaemic conditi oning } \\
\text { versus Minimal invasive esophagectomy without prior gastric is chaemic } \\
\text { conditioning. [Postoperative effects on gastric conduit were asessed] }\end{array}$ \\
\hline 5. & Shen et al & $\begin{array}{c}\text { Minimal access oesophagectomy with low tidal volume vs minimally invasive } \\
\text { esophagectomy with conventional tidal volume } \\
\text { [Postoperative effects on lung complications were assessed] }\end{array}$ \\
\hline 6 & $\begin{array}{l}\text { Cuesta et } \\
\text { al }\end{array}$ & $\begin{array}{l}\text { Immunological changes after minimal invasive oesophagectomy } \\
\text { Versus Immunological changes conventional oesophagectomy }\end{array}$ \\
\hline 7. & Shen et al & $\begin{array}{c}\text { Thoracoscopic esophagectomy in prone versus Thoracoscopic esophagectomy in } \\
\text { decubitus position. [Study assessing how change in Ergonomics of surgery effects } \\
\text { outcomes] }\end{array}$ \\
\hline 8. & $\begin{array}{l}\text { Nozaki et } \\
\quad \text { al }\end{array}$ & $\begin{array}{c}\text { JCOG0502 Thoracoscopic esophagectomy versus open esophagectomy } \\
\text { [Study comparing outcomes \& complications of thoracoscopic esophagectomy } \\
\text { versus open oesophagectomy] }\end{array}$ \\
\hline 9. & $\begin{array}{l}\text { Takeuchi } \\
\text { et al }\end{array}$ & $\begin{array}{l}\text { Minimal invasive esophagectomy with postoperative enteral feeding versus } \\
\text { parenteral feeding [Study comparing postoperative complication outcomes] }\end{array}$ \\
\hline 10 & Peet et al & Quality of life \& late complications after MAO vs after OO \\
\hline 11. & $\begin{array}{l}\text { Yama } \\
\text { moto et al }\end{array}$ & $\begin{array}{l}\text { Thoracoscopic esophagectomy with amino acid administration versus } \\
\text { Thoracoscopic esophagectomy without amino acid adm inistration. } \\
\text { [Study assessing effects on Postop complications] }\end{array}$ \\
\hline$* 12$ & $\begin{array}{l}\text { Mariette et } \\
\text { al }\end{array}$ & $\begin{array}{c}\text { Open versus laparoscopic assisted oesophagectomy } \\
\text { [Study comparing postoperative outcomes] }\end{array}$ \\
\hline
\end{tabular}

\section{STUDY SELECTION}

INCLUSION CRITERIA: RCTs meeting the were:

following criteria were selected:

1.Completed RCTs reporting the MAO outcomes,

2.Completed RCTs reporting the MAO outcomes in comparison with $\mathrm{OO}$ outcomes,

3. Completed RCTs reporting results of peri-operative substances affecting MAO outcome,

4.Completed RCTs comparing different MAO techniques and their outcomes,

5.Completed RCTs reporting alteration MAO outcomes due to ny factor
EXCLUSION CRITERIA: The studies excluded

1.All studies that are not RCTs.

2.Trials reporting MAO outcomes but not randomized.

3.RCTs that fit inclusion criteria (as per their study protocol) but still incomplete.

4.RCTs that discuss oesophagectomy but neither report any MAO outcome nor any factor that directly or indirectly can influence any MAO outcome.

5.RCTs that compare MAO and adjuvant/neoadjuvant chemo/radiotherapy 
HOW THE INCLUSION \& EXCLUSION CRITERIA WERE USED - The refined search had provided total of 796 articles from different databases. This search result was screened by reading titles. At this point abstracts were not read since it was possible by reading the titles only to exclude most of the studies as they were non RCT studies or studies that were trials but irrelevant to the current topic of interest These excluded studies mostly met the exclusion criteria 1 and 2. None of them obviously met the inclusion criteria. The exclusion by reading titles and removing duplicates within each database lead to a list of 57 studies from all databases. The term "57 non-duplicate studies" used in the PRISMA flow-chart above actually means that there remained no duplicates in indivisual database search result and search results of indivisual databases were combined to get this list of 57 articles. The duplicates were however still there in this combined result because the same study could be found in multiple databases. These 57 articles had to be screened again to remove the duplication. The abstracts were read. 24 articles were excluded as they either did not meet all inclusion criteria or they were duplicated in multiple data bases. 13 articles were identified and found to meet the inclusion criteria but when full texts of these studies were read, one article had to be excluded (Appendix 6) because it was a trial (E2202 study) reporting MAO outcomes but not involving the randomisation process. The first inclusion criteria of being a randomised controlled trial was not met. The selected 12 RCTs met either one or multiple inclusion criteria. Table 3 above provides information about what each included RCT reports.

Outcome \& justification of selection process - The search strategy (with the inclusion and exclusion criteria) as described above resulted in a list of 12 RCTs. They provide authent ic information about the outcomes of MAO and factors influencing these outcomes. In evidence based medicine,PICO strategy helps formulation of the research question and aids search for literature(Schardt et al., 2007).

\begin{tabular}{|l|l|l|}
\hline P & Population & $\begin{array}{l}\text { Patients with histologically proven oesophageal cancer who gave } \\
\text { written consent to have minimal access oesophagectomy }\end{array}$ \\
\hline I & Intervention & Minimal access oesophagectomy \\
\hline C & Comparison & Open oesophagectomy \\
\hline O & Outcomes & Post operative outcomes \\
\hline
\end{tabular}

However only 5 of the 12 trials can be seen as comparing MAO \& OO outcomes. In the re maining RCTs, intervention and comparison factors differ although the outcome report ed is one or the other outcome of MAO in every trial. The same outcome was not report ed in all the RCTs identified. So PICO for the remining RCTs is the following

\begin{tabular}{|l|l|l|}
\hline P & Population & $\begin{array}{l}\text { Patients with histologically proven oesophageal cancer who gave } \\
\text { written consent to have minimal access oesophagectomy }\end{array}$ \\
\hline I & Intervention & $\begin{array}{l}\text { Periopertive intervention [administrtion of substance or technique } \\
\text { change] to check effect on MAO }\end{array}$ \\
\hline C & Comparison & NO intervention (CONTROL GROUP) \\
\hline O & Outcomes & Post operative outcomes \\
\hline
\end{tabular}

Critical appraisal : Criteria used to determine quality with justification : This review included RCTs only. The appraisal process to determine the quality of RCTs may involve using tools like the
Cochrane Collaboration Risk of Bias Tool (CCRBT) and Effective Public Health Practice Project Quality Assessment Tool (EPHPP) (Armijo-Olivo et al., 2010).The criteria that determine the quality of any 
RCT include validity of the res ults of that trial and the usefulness of the results to the local population as per CASP (CASP,UK, 2013). Using CCRBT, EPHPP and CASP tools to assess the RCTs selected in this review, it was established that this review involves high quality studies. The results of all RCTs were found to be valid.

-Processes used to appraise studies - involved using the CASP tool first. The CASP tool assessed the quality of the selected trials in terms of whether the results of the trials are valid, what those results are and whether these results can help locally. To give a glo bal rating to each trial the EPHPP quality assessment tool for quantitative studies was used. It helped to appraise the selected studies for quality, rigour and validity. The CCR BT helped to determine the kinds of bias in the trials selected (Appendix 7). Example ; In Bierer et al, 2012 RCT, bias elimination, use of PICO strategy and randomization is sound but no blinding may reduce the quality of findings.

-Outcome of the appraisal process \& how this information is used to inform the synthesis: Each study was reviewed first by CASP tool and then by EPHPP quality assesment tools and finally by CCRBT tool. The outcome of the appraisal process was th at it became possible to determine the quality of studies in terms of bias, design of RCT, any confounding factors, blindedness in the RCTs, data collection methodology used plus any drop outs. The quality assesment process also established that the results of the selected trials are valid, no low quality studies have been incorporated into the current review. This helped to synthesise high quality evidence in favour of MAO when the combining the results of the selected RCTs.

Ethical appraisal :-Processes used to establish ethical aspects of the review : Ethical approval was not needed to conduct this systematic review.

-Principles used to judge ethical quality : The selected trials were checked to con firmed that the ethical approval had been sought to carry out all the RCTs selected in the current review from the respective ethical committees/ boards wherever the trials were conducted. All patients that participated in the selected RCTs had given written informed consent for participation in the trials as needed. The ethical principle of confidentiality has been followed in all the trials. There is no risk of using information of trial participants as the patients have already provided informed consent for all trials involved.

-Outcome of ethical process appraisal: The ethical appraisal process determined that the patients who participated in the trials need not to be contacted for permission as they had all given valid written informed consent.

-Justification for the above : Generally the ethical review of research proposals is con ducted by the

Faculty Research Ethics Panel (FREP) of the university. As this systematic review did not involve any direct patient contact no ethical approval was sought. Howe ver it was ascertained that the included RCTs had the approval of respective ethical co mmittees and that makes this an ethically acceptable study.

Data extraction: The data was extracted in the form of tables [tables 4-7] by only one reviewer as per university assignment guidance. No strategy was needed to manage missing data in this review. As the review was completed by one reviewer only, there is no second reviewer for verification of data involved in this study.

Since the study aimed to assess MAO outcomes any possible risk factor needs to be addressed and therefore information was collected as follows :

1)patient characteristics (age, gender,BMI, ASA grade) to assess that the patient pop ulations undergoing intervention and control were comparable.

2)intraoperative variables (duration of operation, blood loss, anastomotic site, total lymph node retreival) were assessed to compare different MAO techniques as well as fac tors affecting them.

3)histopathological variables (tumour location, tumour histology, resection achieved, tumour stage) were assessed to establish how effectively MAO can be in resectable OCA management.

4)post-operative variables include the primary outcomes and secondary outcomes sought in this review. These variables are defined in the 
DEFINITION TABLE below.

\begin{tabular}{|c|c|c|c|c|c|c|}
\hline \multicolumn{3}{|c|}{ Primary outcomes } & \multicolumn{4}{|c|}{ (2 Post-operative variables) } \\
\hline \multicolumn{3}{|c|}{$\begin{array}{l}\text { Postoperative } \\
\text { pulmonary } \\
\text { infection }\end{array}$} & \multicolumn{4}{|c|}{$\begin{array}{l}\text { Clinical signs and symptoms of pneumonia with } \\
\text { radiological confirmation by chest X-ray or CT scan } \\
\text { plus positive sputum culture } \\
\text { occuring after operation while in hospital or within one month of } \\
\text { surgery. }\end{array}$} \\
\hline \multicolumn{3}{|c|}{ Hospital stay duration } & \multicolumn{4}{|c|}{$\begin{array}{l}\text { Total time patient had to spend in the hospital for and after the } \\
\text { MAO surgery }\end{array}$} \\
\hline \multicolumn{3}{|c|}{ Secondary outcomes } & \multicolumn{4}{|c|}{ (Intra-operative variables) } \\
\hline \multicolumn{3}{|c|}{$\begin{array}{l}\text { Total duration of } \\
\text { surgery }\end{array}$} & \multirow{2}{*}{\multicolumn{4}{|c|}{$\begin{array}{l}\text { Time from skin incision to skin closure } \\
\text { Amount of blood in } \mathrm{ml} \text { that the patient loses during the MAO } \\
\text { operation as a consequence of the surgical procedure. }\end{array}$}} \\
\hline \multirow{2}{*}{\multicolumn{3}{|c|}{$\begin{array}{l}\text { Blood loss } \\
\begin{array}{l}\text { Adequate Lymph node } \\
\text { retreival }\end{array}\end{array}$}} & & & & \\
\hline & & & \multicolumn{4}{|c|}{$\begin{array}{l}\text { In oesophageal cancer , } 15 \text { lymph nodes retreived is generally } \\
\text { considered adequate lymph node retreival (Merkow et al., 2012) }\end{array}$} \\
\hline \multicolumn{3}{|c|}{ Vocal cord paralysis } & \multicolumn{4}{|c|}{ Clinically hoarseness/voice difficulties following surgery } \\
\hline \multicolumn{3}{|c|}{$\begin{array}{l}\text { Anastomotic } \\
\text { complications }\end{array}$} & \multicolumn{4}{|c|}{ Leak or stenosis at conduit site postoperatively } \\
\hline \multicolumn{3}{|c|}{$\begin{array}{l}\text { Quality of life after } 6 \\
\text { weeks post-operatively }\end{array}$} & \multicolumn{4}{|c|}{$\begin{array}{l}\text { Quality of life assessed using EORTC questionnares (Appendix 6) } \\
\text { [European Organization for Research and treatment of cancer] }\end{array}$} \\
\hline & $\begin{array}{l}\text { TRIA } \\
\text { L } \\
\text { author } \\
\text { S }\end{array}$ & $\begin{array}{l}\text { Male } \\
\mathrm{OO} \\
\mathrm{MAO}\end{array}$ & $\begin{array}{l}\text { Female } \\
\mathrm{OO}: \\
\mathrm{MAO}\end{array}$ & $\begin{array}{ll}* & \text { Age } \\
(\text { years) } & \\
\text { OO } & : \\
\text { MAO } & \end{array}$ & $\begin{array}{l}\dagger \mathrm{BMI}(\mathrm{kg} / \mathrm{m} 2) \\
\mathrm{OO}: \mathrm{MAO}\end{array}$ & $\begin{array}{l}\text { ASA GRADE } \\
\text { OO : MAO }\end{array}$ \\
\hline 1 & $\begin{array}{l}\text { Biere } \\
\text { et al }\end{array}$ & $\begin{array}{l}46: 43 \\
82 \%: \\
73 \%\end{array}$ & $\begin{array}{l}10 \\
16 \\
18 \%: \\
27 \%\end{array}$ & $\begin{array}{l}62: 62 \\
(42-75):(34- \\
75)\end{array}$ & $\begin{array}{c}24: 25 \\
(3 \cdot 7) \quad(3 \cdot 6)\end{array}$ & 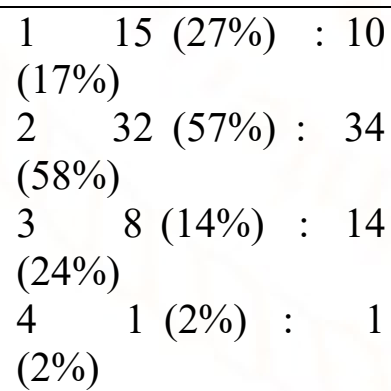 \\
\hline 2 & $\begin{array}{l}\text { Cuesta } \\
\text { et al }\end{array}$ & $12: 10$ & $1: 4$ & $\begin{array}{l}62: 65 \\
(52-74) \\
(56-75)\end{array}$ & $\begin{array}{l}23: 24 \\
(21-33):(16- \\
33)\end{array}$ & APNI \\
\hline 3 & $\begin{array}{l}\text { Peet } \\
\text { et al }\end{array}$ & $\begin{array}{cr}46: & 43 \\
82 \% & : \\
73 \% & \end{array}$ & $\begin{array}{l}10: 16 \\
18 \%: \\
27 \%\end{array}$ & $\begin{array}{l}\quad 62: 62 \\
(42-75):(34- \\
75)\end{array}$ & $\begin{aligned} 24: & 25 \\
(3.7) & (3.6)\end{aligned}$ & 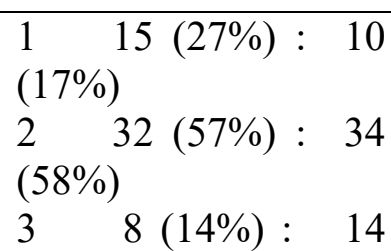 \\
\hline
\end{tabular}


International Journal of Trend in Scientific Research and Development (IJTSRD) ISSN: 2456-6470

\begin{tabular}{|c|c|c|c|c|c|c|}
\hline & & & & & & $\begin{array}{l}(24 \%) \\
4 \quad 1(2 \%): 1(2 \%)\end{array}$ \\
\hline 4 & $\begin{array}{l}\text { Nozak } \\
i \text { et al }\end{array}$ & $\begin{array}{l}93: 82 \\
85.3 \%: 81 \\
2 \\
\mathrm{P}=0.462\end{array}$ & $\begin{array}{l}16: 19 \\
14.7: 18 \\
.8\end{array}$ & $\begin{array}{l}62: 63 \\
(41-75):(48- \\
75) \\
P=0.522 b\end{array}$ & $\begin{array}{l}22: 23 \\
(13-29):(17-28) \\
P=0.934 b\end{array}$ & APNI \\
\hline 5 & $\begin{array}{l}\text { Ito et } \\
\text { al }\end{array}$ & $20: 14$ & $0: 2$ & $\begin{array}{l}58.9 \pm 9.3: \\
61.8 \pm 8.4\end{array}$ & APNI & APNI \\
\hline 6 & $\begin{array}{l}\text { Yama } \\
\text { moto } \\
\text { et al }\end{array}$ & $\begin{array}{l}\text { S:AA } \\
51 / 60\end{array}$ & $\begin{array}{l}\mathrm{S} \\
\text { A.A } \\
9 / 10\end{array}$ & $\begin{array}{r}\mathrm{S}: \mathrm{A} \\
64.56+-8.4: \\
65.66+-7.7 \\
\mathrm{P}=0.94\end{array}$ & $\begin{array}{r}\text { S:AA } \\
21.5+-3.2 \\
21.8+-3.8 \\
\mathrm{P}=0.876\end{array}$ & $\begin{array}{cc}\text { ASA } & \text { S:AA } \\
1 & 13: 12 \\
2 & 22: 20 \\
\mathrm{P}=0.93 & \end{array}$ \\
\hline 7 & $\begin{array}{l}\text { Wajed } \\
\text { et al }\end{array}$ & APNI & APNI & APNI & APNI & APNI \\
\hline 8 & $\begin{array}{l}\text { Shen } \\
\text { et al } \\
\text { PP:DP } \\
\text { 35:32 }\end{array}$ & $\begin{array}{l}\text { PP: DP } \\
26: 24 \\
P=.946 x\end{array}$ & $\begin{array}{l}\text { PP:DP } \\
9: 8\end{array}$ & $\begin{array}{r}\text { PP:DP } \\
60.5+-7.3: \\
60.9+-8.4 \\
P=0.836 t\end{array}$ & \begin{tabular}{l}
\multicolumn{1}{c}{ PP:DP } \\
$23.4+-$ \\
$4.1: 22.7+-3.9$ \\
$\mathrm{P}=0.477 \mathrm{t}$
\end{tabular} & $\begin{array}{rl}\text { ASA } & \text { PP:DP } \\
1 & 13: 12 \\
2 & 22: 20\end{array}$ \\
\hline 9 & $\begin{array}{l}\text { Ninom } \\
\text { iya et } \\
\text { al } \\
\mathrm{C}: \mathrm{S}\end{array}$ & $\begin{array}{l}\mathrm{C}: \mathrm{S} \\
10: 7\end{array}$ & $\begin{array}{l}C: S \\
0: 3\end{array}$ & $\begin{array}{l}C: S \\
63: 64 \\
P=0.91\end{array}$ & $\begin{array}{l}\mathrm{C}: \mathrm{S} \\
\mathrm{APNI}\end{array}$ & $\begin{array}{l}\mathrm{C}: \mathrm{S} \\
\mathrm{APNI}\end{array}$ \\
\hline $\begin{array}{l}1 \\
0\end{array}$ & $\begin{array}{l}\text { Shen } \\
\text { et al } \\
(2013) \\
P V: C \\
\text { V } \\
43: 48\end{array}$ & $\begin{array}{l}\mathrm{PV}: \mathrm{CV} \\
40: 32\end{array}$ & $\begin{array}{l}\mathrm{PV}: \mathrm{CV} \\
13: 16\end{array}$ & $\begin{array}{l}\mathrm{PV}: \mathrm{CV} \\
60.5+7.3: \\
57.2+-9.1 \\
\mathrm{P}=0.403\end{array}$ & $\mathrm{PV}: \mathrm{CV}$ & $\begin{array}{ll}\text { ASA } & \text { PV:CV } \\
& \\
1 & 20: 25 \\
2 & 33: 23\end{array}$ \\
\hline $\begin{array}{l}1 \\
1\end{array}$ & $\begin{array}{l}\text { Takeu } \\
\text { chi } \\
\text { et al }\end{array}$ & $\begin{array}{l}\text { Pn:En } \\
18: 19\end{array}$ & $\begin{array}{c}\text { Pn:En } \\
5: 9\end{array}$ & \begin{tabular}{l}
\multicolumn{1}{c}{ Pn:En } \\
$60.7 \pm 8.97:$ \\
$63.6 \pm 7.13$ \\
$P=0.281$
\end{tabular} & $\begin{array}{l}\text { Pn:En } \\
\text { APNI }\end{array}$ & $\begin{array}{l}\text { Pn:En } \\
\text { APNI }\end{array}$ \\
\hline
\end{tabular}

Table 4a

Patient characteristics (? Risk factors) in RCTs comparing OO \& MAO

The comparison is expressed as ratio in RCT column to fit the table. The abbreviations/signs are explained in text box. 


\begin{tabular}{|l|l|}
\hline $\begin{array}{l}\text { Data includes n (\%), median (range) and } \\
\text { mean (SD). }\end{array}$ & $\mathrm{OO}=$ open oesophagectomy, \\
\hline $\begin{array}{l}\dagger \text { Normal distribution, Independent Samples } \\
\mathrm{t} \text { test applied }\end{array}$ & $\mathrm{MAO}=$ minimal access oesophagectomy. \\
\hline BMI=body-mass index. & $\begin{array}{l}\mathrm{ASA}=\text { American Association } \\
\text { Anesthesiologist }\end{array}$ \\
\hline $\begin{array}{l}\text { *Skewed distribution, Mann-Whitney test } \\
\text { applied }\end{array}$ & $\begin{array}{l}\text { *Sewed distribution, Mann-Whitney test } \\
\text { applied }\end{array}$ \\
\hline $\mathrm{P}=$ fishers exact test & $\mathrm{B}=$ Wilcoxon rank sum test \\
\hline APNI=Authors provide no information & \\
\hline
\end{tabular}

Table 4b Patient characteristics (? Risk factors) in RCTs studying factors affecting MAO outcomes

The factor studied versus control (expressed as ratio) has been abbreviated to fit the table. The abbreviations are explained in the text box and are used in subsequent boxes also

\begin{tabular}{|l|l|}
\hline $\mathrm{DP}=$ Decubitus position oesophagectomy & $\mathrm{PP}=$ prone position oesophagectomy \\
\hline APNI=Authors provide no information & $\begin{array}{c}\mathrm{CV}=\text { Controlled conventional tidal } \\
\text { volume ventilation }\end{array}$ \\
\hline $\mathrm{C}=$ controls receiving saline $\mathrm{x}=\mathrm{x} 2$ test & $\mathrm{S}=$ Sivelestat sodium hydrate group \\
\hline $\mathrm{Pn}=$ Parenteral nutrition & $\mathrm{t}=$ student $\mathrm{t}$-test En= Enteral nutrition \\
\hline $\mathrm{S}: \mathrm{AA}=$ Saline $:$ Amino acid & $\begin{array}{l}\mathrm{PV}=\text { Preserved low tidal volume } \\
\text { ventilation }\end{array}$ \\
\hline $\mathrm{Nm}=$ Neoadjuvant therapy+MAO & Om=Only MAO $\mathrm{m}=\mathrm{MAO}$ \\
\hline $\mathrm{N}+\mathrm{m}$-neoadjuvant treatment $+\mathrm{MAO}$ & \\
\hline
\end{tabular}

Table 5a Histopathological characteristics tumours

\begin{tabular}{|c|c|c|c|c|c|}
\hline RCT authors & Biere et al & Cuesta et al & Peet et al & Ito et al & Nozaki et al \\
\hline & OO:MAO & OO:MAO & OO:MAO & OO:MAO & OO:MAO \\
\hline $\begin{array}{l}\text { Tumour locat } \\
\text { ion } \\
\text { oesophagus } \\
\text { Upper } \\
\text { Middle } \\
\text { Lower }\end{array}$ & $\begin{array}{cc}3 & : 1 \\
22: 26 & \\
31: 32 & \end{array}$ & APNI & $\begin{array}{l}3: 1 \\
22: 26 \\
31: 32\end{array}$ & APNI & $\begin{array}{l}12: 15 p=0.18 \\
65: 59.6 \\
32: 19\end{array}$ \\
\hline
\end{tabular}


International Journal of Trend in Scientific Research and Development (IJTSRD) ISSN: 2456-6470

\begin{tabular}{|c|c|c|c|c|c|}
\hline $\begin{array}{l}\text { Tumour type } \\
\text { ACA } \\
\text { SCCA } \\
\text { Others }\end{array}$ & $\begin{array}{l}36: 35 \\
19: 24 \\
1: 0\end{array}$ & $\begin{array}{l}11: 13 \mathrm{p}=\mathrm{ns} \\
2: 1 \mathrm{p}=\mathrm{ns}\end{array}$ & $\begin{array}{l}36: 35 \\
19: 24 \\
1: 0\end{array}$ & APNI & APNI \\
\hline $\begin{array}{l}\text { Noeoadjuvant } \\
\text { Chemoradioth } \\
\text { Chemoth }\end{array}$ & $\begin{array}{l}52: 54 \\
4: 5\end{array}$ & APNI & $\begin{array}{l}52: 54 \\
4: 5\end{array}$ & APNI & $\begin{array}{l}\text { Definitive } \\
\text { ChemoRTH } \\
\text { 159: }\end{array}$ \\
\hline $\begin{array}{l}\text { STAGE } \\
0 \\
\text { I } \\
\text { IIa } \\
\text { IIb } \\
\text { III } \\
\text { IV }\end{array}$ & $\begin{array}{l}0: 1 \\
4: 4 \\
16: 17 \\
6: 9 \\
14: 11 \\
5: 4\end{array}$ & APNI & $\begin{array}{l}0: 1 \\
4: 4 \\
16: 17 \\
6: 9 \\
14: 11 \\
5: 4\end{array}$ & APNI & APNI \\
\hline $\begin{array}{ll}\text { No } & \text { residual } \\
\text { nodes } & \end{array}$ & $7: 9$ & APNI & $7: 9$ & APNI & APNI \\
\hline $\begin{array}{l}\text { Resection } \\
\text { margin } \\
\text { R0 } \\
\text { R1 }\end{array}$ & $\begin{array}{l}47: 54 \\
5: 1\end{array}$ & APNI & $\begin{array}{l}47: 54 \\
5: 1\end{array}$ & APNI & APNI \\
\hline
\end{tabular}

\begin{tabular}{|c|c|c|c|c|c|c|}
\hline RCT authors & $\begin{array}{l}\text { Yamam } \\
\text { ato et al }\end{array}$ & $\begin{array}{l}\text { Waje } \\
\text { d et } \\
\text { al }\end{array}$ & $\begin{array}{l}\text { Shen et } \\
\text { al }(2014)\end{array}$ & $\begin{array}{l}\text { Takeuch } \\
\text { i et al }\end{array}$ & $\begin{array}{l}\text { Shen et } \\
\text { al } \\
(2013)\end{array}$ & $\begin{array}{l}\text { Ninomiy } \\
\text { a et al }\end{array}$ \\
\hline & $\mathrm{S}: \mathrm{AA}$ & & PP:DP & Pn:en & $\mathrm{PV}: \mathrm{CV}$ & $\mathrm{C}: \mathrm{S}$ \\
\hline $\begin{array}{l}\text { Tumour location } \mathrm{U} \\
\text { In M esophagus LOG }\end{array}$ & APNI & $\begin{array}{l}\text { APN } \\
\text { I }\end{array}$ & $\begin{array}{l}6: 5 \\
22: 20 \\
7: 7\end{array}$ & APNI & $\begin{array}{l}7: 10 \\
38: 31 \\
8: 7\end{array}$ & $\begin{array}{l}3: 2 \\
4: 5 \\
3: 4\end{array}$ \\
\hline
\end{tabular}


International Journal of Trend in Scientific Research and Development (IJTSRD) ISSN: 2456-6470

\begin{tabular}{|c|c|c|c|c|c|c|}
\hline $\begin{array}{l}\text { Tumour type } \\
\text { ACA } \\
\text { SCCA } \\
\text { Others }\end{array}$ & APNI & $\begin{array}{l}\text { APN } \\
\text { I }\end{array}$ & $\begin{array}{l}2: 3 \\
33: 29 \\
p=0.917\end{array}$ & APNI & $\begin{array}{l}3: 2 \\
50: 46 \\
P=.909\end{array}$ & APNI \\
\hline $\begin{array}{l}\text { Noeoadjuvant } \\
\text { Treatment } \\
\text { Chemoradioth } \\
\text { Chemoth }\end{array}$ & APNI & $\begin{array}{l}\text { APN } \\
\text { I }\end{array}$ & APNI & $\begin{array}{l}P=0.658 \\
13: 12\end{array}$ & APNI & APNI \\
\hline $\begin{array}{l}\text { STAGE } \\
0 \\
\text { I } \\
\text { IIa } \\
\text { IIb } \\
\text { III } \\
\text { IV }\end{array}$ & $\begin{array}{l}\mathrm{P}=0.72 \\
33: 40 \\
13: 9 \\
14: 21 \\
0: 0\end{array}$ & $\begin{array}{l}\text { APN } \\
\text { I }\end{array}$ & APNI & $\begin{array}{l}\mathrm{P}=0.469 \\
11: 10 \\
7: 6 \\
3: 4 \\
2: 4\end{array}$ & $\begin{array}{l}\mathrm{P}=.86 \\
7: 5 \\
11: 9 \\
35: 34\end{array}$ & $\begin{array}{l}\mathrm{P}=0.572 \\
0: 0 \\
4: 2 \\
4: 5 \\
0: 3 \\
2: 0\end{array}$ \\
\hline $\begin{array}{l}\text { No residual } \\
\text { Lymph nodes }\end{array}$ & APNI & APN & APNI & APNI & APNI & APNI \\
\hline $\begin{array}{l}\text { Resection margins } \\
\text { R0 } \\
\text { R1 }\end{array}$ & APNI & $\begin{array}{l}\text { APN } \\
\text { I }\end{array}$ & APNI & APNI & APNI & APNI \\
\hline
\end{tabular}

Table 5b- Histopathological characteristics tumours

The tables hereafter only contain mean/median values. This was done to simplify the ratios. The range/2SD are given in the RCTs for some of these variables.

Table 6a Intraoperative data of the studies comparing OO:MAO

\begin{tabular}{|c|c|c|c|c|}
\hline $\begin{array}{l}\text { Intra- } \\
\text { operative } \\
\text { data }\end{array}$ & $\begin{array}{l}\text { Biere et } \\
\text { al } \\
\text { OO:M } \\
\mathrm{AO}\end{array}$ & $\begin{array}{l}\text { Cuesta et } \\
\text { al } \\
\text { OO:MAO }\end{array}$ & $\begin{array}{l}\text { Peet et } \\
\text { al } \\
\mathrm{OO}: \mathrm{M} \\
\mathrm{AO}\end{array}$ & $\begin{array}{l}\text { Nozaki et al } \\
\text { OO:MAO }\end{array}$ \\
\hline $\begin{array}{l}\text { Surgery time } \\
\text { (in }\end{array}$ & 299:32 & $266: 305$ & 299:32 & $399: 510$ \\
\hline
\end{tabular}




\begin{tabular}{|l|l|l|l|l|}
\hline minutes)*t & 9 & $\mathrm{P}=\mathrm{ns}$ & 9 & $\mathrm{P}<.001 \mathrm{~b}$ \\
\hline $\begin{array}{l}\text { Blood } \\
\text { loss (in mL) } \mathrm{t}\end{array}$ & $\begin{array}{l}45: 200 \\
\mathrm{P}<0.00 \\
1\end{array}$ & $\begin{array}{l}\mathrm{P}=.045 \\
2\end{array}$ & $\begin{array}{l}\mathrm{P}<0.00 \\
1\end{array}$ & $\mathrm{P}<.001 \mathrm{~b}$ \\
\hline $\begin{array}{l}\text { Anastomotic } \\
\text { Level } \\
\text { cervical }\end{array}$ & $37: 38$ & $\mathrm{APNI}$ & $37: 38$ & $\mathrm{APNI}$ \\
- thoracic & $15: 17$ & $15: 17$ & \\
\hline $\begin{array}{l}\text { Total lymph } \\
\text { nodes } \\
\text { retreived }\end{array}$ & $21: 20$ & $19: 19$ & $21: 20$ & $47: 56$ \\
\hline
\end{tabular}

\begin{tabular}{|l|l|}
\hline $\mathrm{s}=$ student $\mathrm{t}$-test & $\mathrm{b}=$ Wilcoxon rank sum test \\
\hline *-time from skin incision to closure, & $\mathrm{t}=$ skewed distribution, \\
\hline $\begin{array}{l}\text { Data includes median (range) or } \\
\text { mean (SD) }\end{array}$ & \\
\hline
\end{tabular}

Table 6b Intraoperative data in studies on factors affecting MAO outcomes

\begin{tabular}{|c|c|c|c|c|c|}
\hline $\begin{array}{l}\text { Intra- } \\
\text { operativ } \\
\text { e } \\
\text { data }\end{array}$ & $\begin{array}{l}\text { Yamamo } \\
\text { to et al } \\
\text { S:AA }\end{array}$ & $\begin{array}{l}\text { Shen et } \\
\text { al (2013) } \\
\text { CV:PV }\end{array}$ & $\begin{array}{l}\text { Ninomiy } \\
\text { a et al } \\
\text { C:S }\end{array}$ & $\begin{array}{l}\text { Shen et al } \\
(2014) \\
\text { PP:DP }\end{array}$ & $\begin{array}{l}\text { Takeuchi } \\
\text { et al } \\
(2015) \\
\text { Pn : En }\end{array}$ \\
\hline $\begin{array}{l}\text { Surgery } \\
\text { Time } \\
\text { (minutes } \\
\text { ) }\end{array}$ & $\begin{array}{l}374: 365 \\
P=0.29\end{array}$ & $\begin{array}{l}214: 195 . \\
8 \\
\mathrm{P}=0.338 \\
*\end{array}$ & $\begin{array}{l}549: 517 \\
P=0.393\end{array}$ & $\begin{array}{ll}\text { Th } 68 & : 87 \\
\text { p }<.001 \mathrm{~s} & \\
\text { Ab 55: } & 51, \\
\text { p.397 } & \end{array}$ & $\begin{array}{l}548: 564 \\
P=0.395\end{array}$ \\
\hline $\begin{array}{l}\text { Blood } \\
\text { loss } \\
(\mathrm{mL})\end{array}$ & $\begin{array}{l}210.2: 16 \\
8.8 \\
P=0.75\end{array}$ & $\begin{array}{l}130: 170 \\
P=.728 t\end{array}$ & $\begin{array}{l}320: 305 \\
P=0.796\end{array}$ & $89: 67 \mathrm{p}<.001 \mathrm{~s}$ & $\begin{array}{l}211: 20 \\
P=0.616\end{array}$ \\
\hline $\begin{array}{l}\text { Anastom } \\
\text { osis } \\
\text { level }\end{array}$ & APNI & APNI & APNI & APNI & APNI \\
\hline Total & APNI & APNI & APNI & $18.2: 15.4$ & $67: 77$ \\
\hline
\end{tabular}




\begin{tabular}{|l|l|l|l|l|l|}
\hline $\begin{array}{l}\text { lymph } \\
\text { nodes } \\
\text { retreived }\end{array}$ & & & & $\mathrm{P}<.001 \mathrm{~s}$ & $\mathrm{P}=0.053$ \\
\hline
\end{tabular}

Table 6c Intraoperative data in studies on factors affecting MAO outcomes

\begin{tabular}{|c|c|c|}
\hline & $\begin{array}{l}\text { Trial } \\
\text { authors }\end{array}$ & \\
\hline 1. & Ito et al & $\begin{array}{l}\text { The intra-operative details in this trial are: } \\
\qquad \text { OO: MAO } \\
\text { Vital capacity (1) } \quad 3.75: 3.50 \\
\text { Vital capacity (\%) } \quad 109.3: 107.8 \\
\text { FEV1 } 2.88: 2.60 \\
\text { FEV1(\%) } 81.0: 82.3 \\
\text { Peak expiratory flow (1/s) } 7.95: 7.08 \\
\text { \{only mean values given here } \text {-no statistically differences noted by } \\
\text { authors }\end{array}$ \\
\hline 2. & Wajed et al & Only data about conduit perfusion is available in this trial. \\
\hline
\end{tabular}

Table 7a- Postoperative data

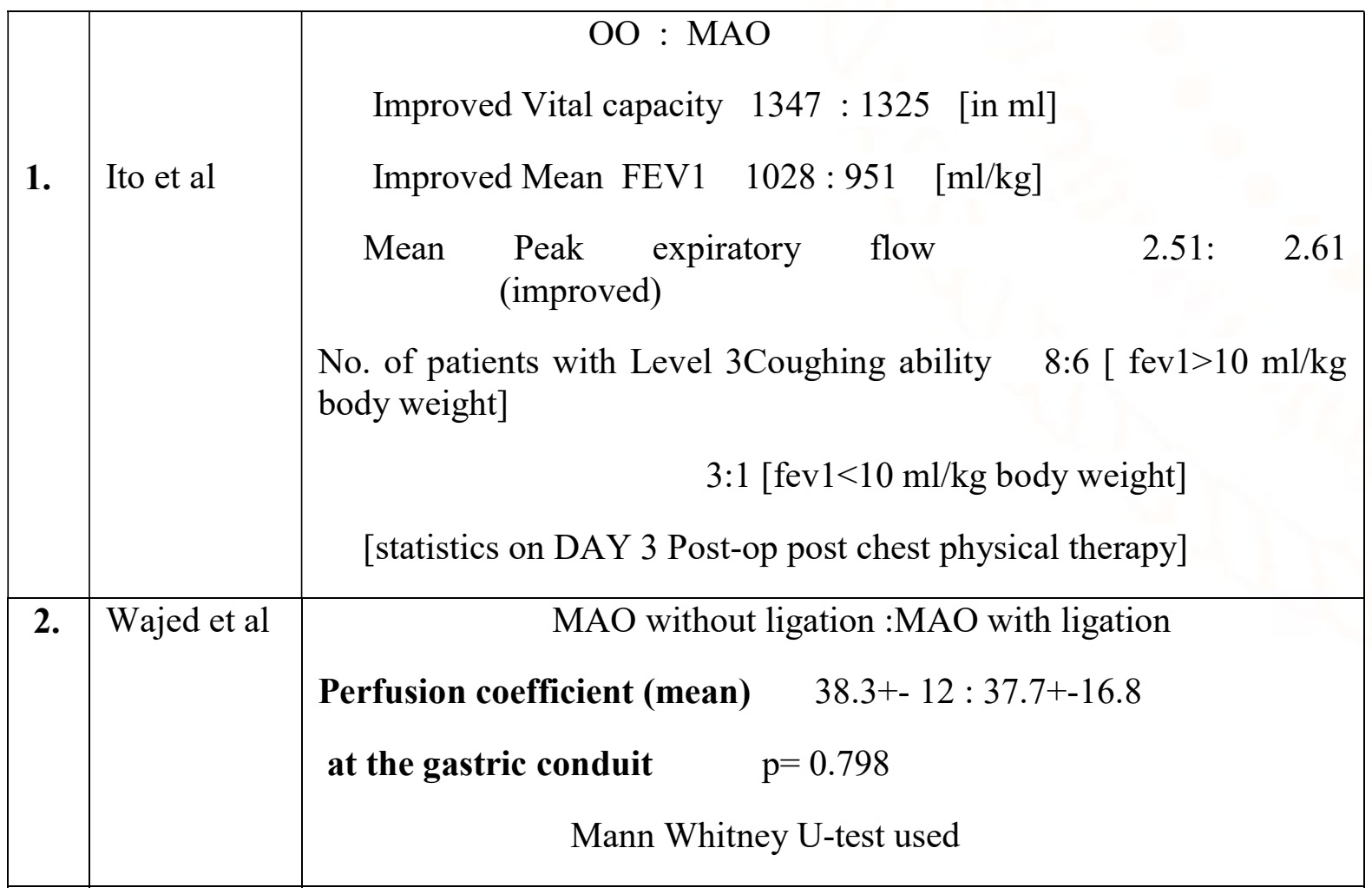




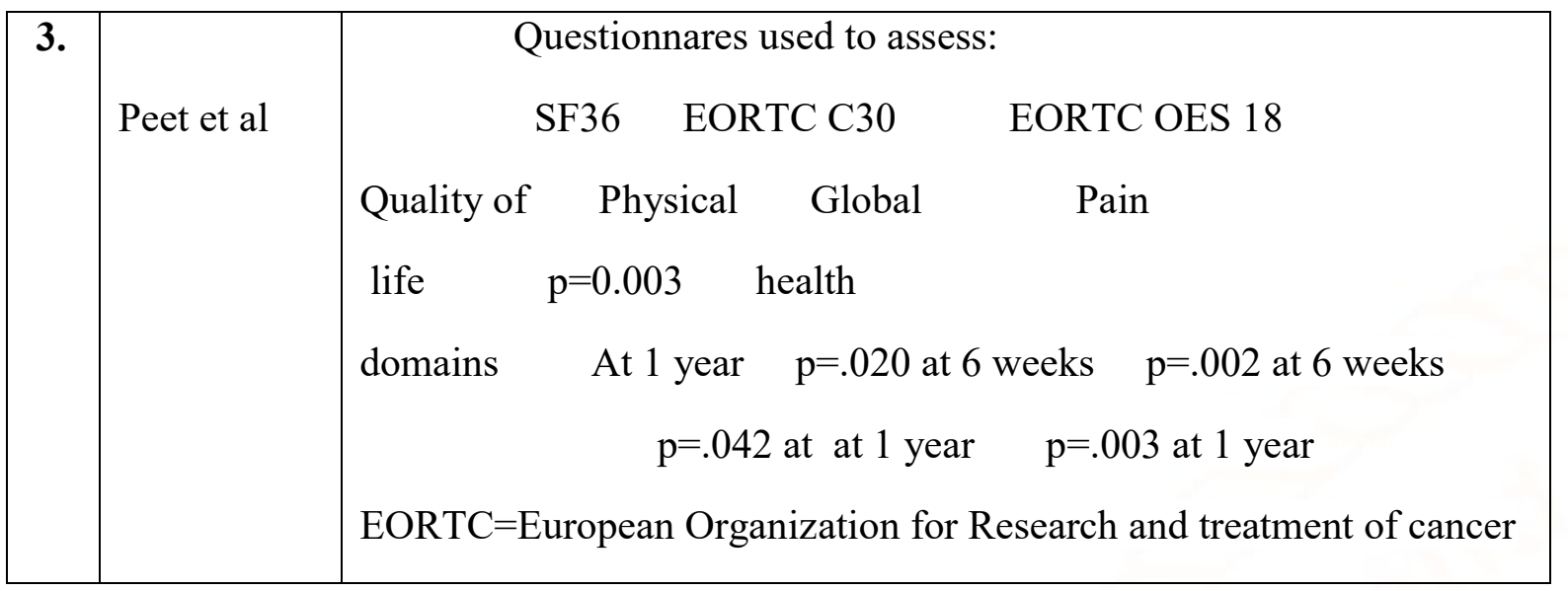

TABLE 7B Post-operative Data

\begin{tabular}{|c|c|c|c|c|}
\hline & $\begin{array}{l}\text { Biere et al } \\
\text { OO:MAOp } \\
\text { value }\end{array}$ & $\begin{array}{l}\text { Cuesta } \\
\text { et al } \\
\text { OO:M } \\
\text { AOpva } \\
\text { lue }\end{array}$ & $\begin{array}{l}\text { Nozaki et al } \\
\text { OO:MAO } \\
\text { pvalue }\end{array}$ & $\begin{array}{l}\text { Ninomiya } \\
\text { et al } \\
\mathrm{C}: \mathrm{S}\end{array}$ \\
\hline $\begin{array}{l}\text { ICU stay [days] } \\
t\end{array}$ & $\begin{array}{l}1: 1 \\
P=0.06\end{array}$ & $\begin{array}{l}1: 1 \\
\mathrm{P}=\mathrm{ns}\end{array}$ & - & $\begin{array}{l}3.10: 2.66 \\
P=0.481\end{array}$ \\
\hline Length of stay & - & - & $24: 24 p=0.472$ & $31: 32 p=0.853$ \\
\hline $\begin{array}{l}\text { VAS [10 DAYS }] \\
\text { L }\end{array}$ & $\begin{array}{l}3: 2 \\
\mathrm{P}=0.001\end{array}$ & APNI & - & APNI \\
\hline Epidural failure & $\begin{array}{l}11: 10 \\
\mathrm{P}=0.734\end{array}$ & APNI & - & APNI \\
\hline $\begin{array}{l}\text { Pulmonary } \\
\text { infection } \\
\text { hospital in }\end{array}$ & $\begin{array}{l}19: 7 \\
P=0.005\end{array}$ & $\begin{array}{l}\text { 1:0 } \\
\mathrm{p}=\mathrm{ns} \\
\text { Empye } \\
\mathrm{ma}\end{array}$ & $\begin{array}{l}24: 11 \quad \mathrm{p}=.041 \\
\text { Atelectasis }\end{array}$ & $\begin{array}{l}1: 1 \\
\{\text { PNEMONIA }\}\end{array}$ \\
\hline $\begin{array}{l}\text { Pulmonary } \\
\text { embolism }\end{array}$ & $\begin{array}{l}0: 1 \\
P=0.328\end{array}$ & $\begin{array}{l}0: 1 \\
\mathrm{P}=\mathrm{ns}\end{array}$ & & APNI \\
\hline $\begin{array}{l}\text { Anastomotic } \\
\text { leakage }\end{array}$ & $\begin{array}{l}4: 7 \\
P=0.390\end{array}$ & $\begin{array}{l}1: 3 \\
p=n s\end{array}$ & $\begin{array}{l}15: 7 \\
P=0.12\end{array}$ & APNI \\
\hline $\begin{array}{l}\text { Other } * * \\
\text { complications }\end{array}$ & $\begin{array}{l}2: 2 \\
\mathrm{P}=0.958\end{array}$ & APNI & $\begin{array}{lr}4: 2 & \mathrm{p}=0.684 \\
\text { intravascular } & \text { catheter } \\
\text { infection } & \\
2: 3 \quad \mathrm{p}=0.673 & \text { paralytic } \\
\text { ileus } 0: 4 & \mathrm{p}=.052 \\
\text { intestinal } & \text { obstruction }\end{array}$ & $\begin{array}{l}\text { SIRS } \\
49: 17 \\
\mathrm{P}=0.009\end{array}$ \\
\hline
\end{tabular}




\begin{tabular}{|c|c|c|c|c|c|c|c|}
\hline & & & & \multicolumn{2}{|c|}{$\begin{array}{l}5: 16 \quad \mathrm{p}=.01 \quad \text { Other } \\
\text { complications }\end{array}$} & & \\
\hline $\begin{array}{ll}\text { Vocal cord } \\
\text { paralysis e }\end{array}$ & \multicolumn{2}{|c|}{$\begin{array}{l}8: 1 \\
P=0.012\end{array}$} & $\begin{array}{l}2: 1 \\
\mathrm{P}=\mathrm{ns}\end{array}$ & \multicolumn{2}{|c|}{$\begin{array}{l}\text { 17:15 } \quad \mathrm{P}=1 \quad \text { recurrent } \\
\text { nerve paralysis }\end{array}$} & \multicolumn{2}{|c|}{$\begin{array}{l}\mathrm{U} / \mathrm{L} 3: 3 \\
\mathrm{~B} / \mathrm{L} 6: 5\end{array}$} \\
\hline Re-operations & \multicolumn{2}{|c|}{$\begin{array}{l}6: 8 \\
P=0.641\end{array}$} & APNI & $\begin{array}{l}2: 10 \\
P=.016\end{array}$ & & \multicolumn{2}{|c|}{ APNI } \\
\hline 30-day mortality & $0: 1$ & & $\begin{array}{l}0: 1 \\
\mathrm{P}=\mathrm{ns}\end{array}$ & \multicolumn{2}{|l|}{-} & \multicolumn{2}{|c|}{ APNI } \\
\hline $\begin{array}{l}\text { In-hospital } \\
\text { mortality }\end{array}$ & \multicolumn{2}{|c|}{$1: 2$} & & \multicolumn{2}{|l|}{$1: 1 \mathrm{p}=1$} & \multicolumn{2}{|c|}{ APNI } \\
\hline & & & & & & \multicolumn{2}{|c|}{ U/L Unilateral } \\
\hline \multicolumn{3}{|c|}{$\begin{array}{l}\text { ** means those thoracic } \\
\text { complic ations not related to } \\
\text { mediastinitis, empyema, hiatus } \\
\text { hernia, }\end{array}$} & \multicolumn{2}{|c|}{$\begin{array}{l}\mathrm{L}=\text { Linear mixed } \\
\text { model } \mathrm{P}=\mathrm{p} \text { value } \\
\mathrm{B} / \mathrm{L} \text { Bilateral }\end{array}$} & $\begin{array}{l}\text { t-Skewed } \\
\text { distribution }\end{array}$ & \multicolumn{2}{|c|}{$\begin{array}{l}\mathrm{P} \text { value } \\
\text { calculated } \\
\text { byFishers exact } \\
\text { test in Nozaki et } \\
\text { al study }\end{array}$} \\
\hline \multicolumn{3}{|c|}{$\begin{array}{l}\text { chylous leak needing re- } \\
\text { operation }\end{array}$} & \multicolumn{2}{|c|}{$\mathrm{e}=$ endoscopically } & confirmed & \multicolumn{2}{|c|}{$\begin{array}{l}\mathrm{ns}=\text { not } \\
\text { significant }\end{array}$} \\
\hline \multicolumn{2}{|c|}{$\begin{array}{l}\text { Table 7c } \\
\text { operative }\end{array} \begin{array}{r}\text { Post- } \\
\text { data }\end{array}$} & \multicolumn{2}{|c|}{$\begin{array}{l}\text { Shen et al } \\
(2013) \\
\text { PV:CV }\end{array}$} & $\begin{array}{l}\text { Takeuchi } \\
\text { et al } \\
\text { Pn:En }\end{array}$ & \multicolumn{2}{|c|}{$\begin{array}{l}\text { Shen et al } \\
(2014) \\
\text { DP:PP }\end{array}$} & $\begin{array}{l}\text { Yamamoto } \\
\text { et al } \\
\text { S:AA }\end{array}$ \\
\hline \multicolumn{2}{|l|}{$\begin{array}{l}\text { Rate of weight loss } \\
\text { at day } 14 \text { Postop }\end{array}$} & & & $\begin{array}{l}5.05: 2.94 \\
\mathrm{P}=0.02\end{array}$ & \multicolumn{2}{|l|}{ APNI } & APNI \\
\hline \multicolumn{2}{|l|}{ Pneumonia } & \multicolumn{2}{|c|}{$\begin{array}{l}\text { 5: } 13 \quad \mathrm{P}=.021 \\
\text { Pulmonary } \\
\text { complications }\end{array}$} & $7: 3 \quad p=0.137$ & \multicolumn{2}{|c|}{$\begin{array}{l}2: 4 \mathrm{p}=0.587 \\
\text { [pulmonary } \\
\text { Complication } \\
\text { ] }\end{array}$} & $\begin{array}{l}\quad 18: 10 \\
\mathrm{p}=.029 \\
\text { Surgical } \\
\text { Infectious } \\
\text { complicatio } \\
\mathrm{ns}\end{array}$ \\
\hline \multicolumn{2}{|l|}{ Atelectasis } & & & \multirow[t]{2}{*}{$2: 2 \mathrm{p}=0.965$} & \multicolumn{2}{|c|}{ See above } & \\
\hline \multicolumn{2}{|l|}{$\begin{array}{l}\text { Recurrent laryngeal } \\
\text { nerve palsy }\end{array}$} & $\begin{array}{l}4: 13 p \\
\text { Hoarsene }\end{array}$ & $\begin{array}{l}=0.891 \\
\text { ess }\end{array}$ & & $\begin{array}{l}3: 2 \mathrm{p}=0.9 \\
\text { [hoarsene }\end{array}$ & & $\begin{array}{l}13: 6 \\
\mathrm{p}=0.86 \\
\text { Non- } \\
\text { infectious } \\
\text { surgical } \\
\text { complicatio } \\
\text { ns }\end{array}$ \\
\hline
\end{tabular}


International Journal of Trend in Scientific Research and Development (IJTSRD) ISSN: 2456-6470

\begin{tabular}{|l|l|l|l|l|}
\hline Anastomotic leak & $7: 5 \mathrm{P}=.952$ & $4: 5 \mathrm{p}=0.767$ & $3: 3 \mathrm{p}=0.754 \mathrm{f}$ & \\
\hline Postop hospital stay & & $\begin{array}{l}27.1: 28.3 \\
\mathrm{P}=0.147\end{array}$ & APNI & \\
\hline ICU stay & & & APNI & \\
\hline Mortality & $0: 0$ & & $0: 0$ & $0: 0$ \\
\hline
\end{tabular}

$\mathrm{F}=$ fisher exact test

\begin{tabular}{|l|l|}
\hline & RCT specific post-operative \\
\hline Shen et al & $\begin{array}{l}\text { Ergonomic Eye blink rate DP:PP } \mathrm{p}<0.001 \text { for R2 and R1-R2 } \\
\text { Evaluation MSS scores DP:PP } \mathrm{p}<0.001 \\
\mathrm{R}=\text { rate of eye blink/mniute of surgeon at beginning [R1] \& end [R2] }\end{array}$ \\
\hline $\begin{array}{l}\text { Shen et al } \\
{[2013]}\end{array}$ & $\begin{array}{l}\text { Pulmonary complications are significantly lower in the group that receive } \\
\text { lower tidal volume plus PEEP than those who receive conventional tidal } \\
\text { volume }\end{array}$ \\
\hline
\end{tabular}

\begin{tabular}{|l|c|c|}
\hline Mariette et al, 2016 * & $\begin{array}{l}\text { Open } \\
\text { oesophagectomy } \\
104\end{array}$ & $\begin{array}{c}\text { Hybrid minimal invasive } \\
\text { oesophagectomy } \\
103\end{array}$ \\
\hline Major post-operative morbidity $\mathrm{p}=.0001$ & 67 & 37 \\
\hline Major pulmonary complication $\mathrm{p}=.03$ & 31 & 18 \\
\hline 30 day mortality & 5 & 5 \\
\hline
\end{tabular}

Data analysis - The data is quantitative and therefore statistics software was needed. Factors affecting the MAO outcomes were found to be independant and this made it impossible to analyze all the data together in a meta-analysis. The meta-analysis was possible for sub-group of few trials in which there was direct comparison of $\mathrm{MAO}$ and $\mathrm{OO}$ outcomes. In order to measure variability in the data sets obtained from the RCTs, standard deviation was first calculated for all patient numbers in the selected RCTs using online statistics calculators(table below).

STANDARD DEVIATION OF MEAN \& CONFIDENCE INTERVALS

\begin{tabular}{|l|l|l|l|l|l|l|l|l|l|l|}
\hline & & I & C & T & M & SD & $\begin{array}{l}\text { Pop } \\
\text { u } \\
\text { SD }\end{array}$ & $\begin{array}{l}\text { V M } \\
\text { SD }\end{array}$ & $\begin{array}{l}\text { V } \\
\text { popu } \\
\text { SD }\end{array}$ & CI \\
\hline 1 & Biere et al & 59 & 56 & 115 & $\begin{array}{l}57 . \\
5\end{array}$ & 2.12 & 1.5 & 4.5 & 2.25 & 0.39 \\
\hline 2 & Cuesta et al & 14 & 13 & 27 & $\begin{array}{l}13 . \\
5\end{array}$ & 0.71 & 0.5 & 0.5 & 0.25 & 0.27 \\
\hline
\end{tabular}


International Journal of Trend in Scientific Research and Development (IJTSRD) ISSN: 2456-6470

\begin{tabular}{|l|l|l|l|l|l|l|l|l|l|l|}
\hline 3 & Peet et al & 59 & 56 & 115 & $\begin{array}{l}57 . \\
5\end{array}$ & 2.12 & 1.5 & 4.5 & 2.25 & 0.39 \\
\hline 4 & $\begin{array}{l}\text { Nozaki et } \\
\text { al }\end{array}$ & 101 & $\begin{array}{l}10 \\
9\end{array}$ & 210 & 105 & 5.66 & 4 & 32 & 16 & 0.77 \\
\hline 5 & $\begin{array}{l}\text { Yamamoto } \\
\text { Et al }\end{array}$ & 70 & 60 & 130 & 65 & 7.07 & 5 & 50 & 25 & 1.22 \\
\hline 6 & $\begin{array}{l}\text { Shen et al } \\
(2013)\end{array}$ & 53 & 48 & 101 & $\begin{array}{l}50 . \\
5\end{array}$ & 3.53 & 2.5 & 125 & 6.25 & 0.69 \\
\hline 7 & $\begin{array}{l}\text { Ninomiya } \\
\text { et al }\end{array}$ & 11 & 11 & 22 & 11 & 0 & 0 & 0 & 0 & - \\
\hline 8 & $\begin{array}{l}\text { Shen et al } \\
(2014)\end{array}$ & 35 & 32 & 67 & $\begin{array}{l}33 . \\
5\end{array}$ & 2.12 & 1.5 & 4.5 & 2.25 & 0.51 \\
\hline 9 & $\begin{array}{l}\text { Takeuchi } \\
\text { etal }\end{array}$ & 24 & 23 & 47 & $\begin{array}{l}23 . \\
5\end{array}$ & 0.71 & 0.5 & 0.5 & 0.25 & 0.2 \\
\hline 10 & Ito et al & 14 & 22 & 36 & 18 & 5.66 & 4 & 32 & 16 & 1.85 \\
\hline 11 & Wajed et al & 8 & 8 & 16 & 8 & 0 & 0 & 0 & 0 & - \\
\hline$*$ & $\begin{array}{l}\text { Marriette et } \\
\text { al }\end{array}$ & 103 & $\begin{array}{l}10 \\
4\end{array}$ & 207 & $\begin{array}{l}103 \\
.5\end{array}$ & 0.71 & 0.25 & 0.5 & 0.5 & 0.2 \\
\hline
\end{tabular}

Calculations through online statistics calculators [easycalculation.com/statistics/standarddeviation.php/](mccallum-layton.co.uk/tools/statistic-calculators)

\begin{tabular}{|l|l|}
\hline $\begin{array}{l}\text { ABBREVIATIONS USED } \\
\text { above }\end{array}$ & $\begin{array}{l}\mathrm{M}=\text { Mean No-number, C=Control,CI= confidence } \\
\text { interval }\end{array}$ \\
\hline $\begin{array}{l}\text { V= Variance R= Range for the } \\
\text { true popu } \mathrm{M}\end{array}$ & SD=Standard deviation of mean upto 2 decimal \\
\hline $\begin{array}{l}\text { popu=Population } \\
\text { I=Intervention }\end{array}$ & $\begin{array}{l}\text { Secondary outcomes } \\
\text { Primary outcomes } \\
\begin{array}{l}\text { Postoperative pulmonary } \\
\text { infection (PPI) Hospital stay } \\
\text { duration ( HSD) }\end{array}\end{array} \begin{array}{l}\text { lymph node retreival= LNR, Blood loss=BL, } \\
\text { paralysis=VCP, } \\
\text { complications=AC }\end{array}$ \\
\hline
\end{tabular}

Methods used for analysis with justification:To integrate the quantitative findings from similar although separate RCTs, meta-analysis was performed. Continuous measure meta -analysis was used because range was available in the data of others besides the mean value of variable. In this review, the meta-analyses involve only few RCTs because the out comes and intervention/control were comparable only in few RCTs. Where range of variable was given the standard deviation of variable was used based on the formula [that is standard deviation is approximately highest value minus lowest value divided by 4 (Taylor,2016)]. In RCTS without range of variable and only mean values, Odds ratio meta-analysis was performed. MedCalc software was used for meta-ana lysis of all primary and secondary outcomes as it is easy to install plus reliable to use. A forest plot and table for each outcome analyzed was generated during the meta-analysis as seen below. 
Postoperative pulmonary infection

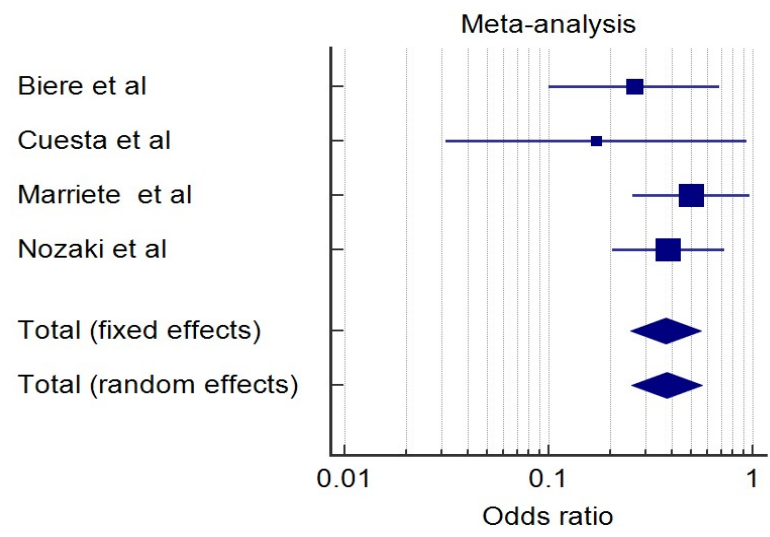

\begin{tabular}{cccc}
\multicolumn{2}{c}{ 1. Intervention groups } & \multicolumn{2}{c}{ 2. Control groups } \\
$\begin{array}{c}\text { Variable for total } \\
\text { number of cases }\end{array}$ & MAO & $\begin{array}{c}\text { Variable for total number } \\
\text { of cases }\end{array}$ & OO \\
$\begin{array}{c}\text { Variable for } \\
\text { number of } \\
\text { positive cases }\end{array}$ & PPI & $\begin{array}{c}\text { Variable for number of } \\
\text { positive cases }\end{array}$ & OOPPI
\end{tabular}

Test for heterogeneity

Q

DF

Significance level

$I^{2}$ (inconsistency)

95\% CI for $\mathrm{I}^{2}$

$$
2.0812
$$

3

$$
\begin{gathered}
P=0.5557 \\
0.00 \% \\
0.00 \text { to } \\
81.39
\end{gathered}
$$

$\mathrm{OO}$

OPPI 
International Journal of Trend in Scientific Research and Development (IJTSRD) ISSN: 2456-6470

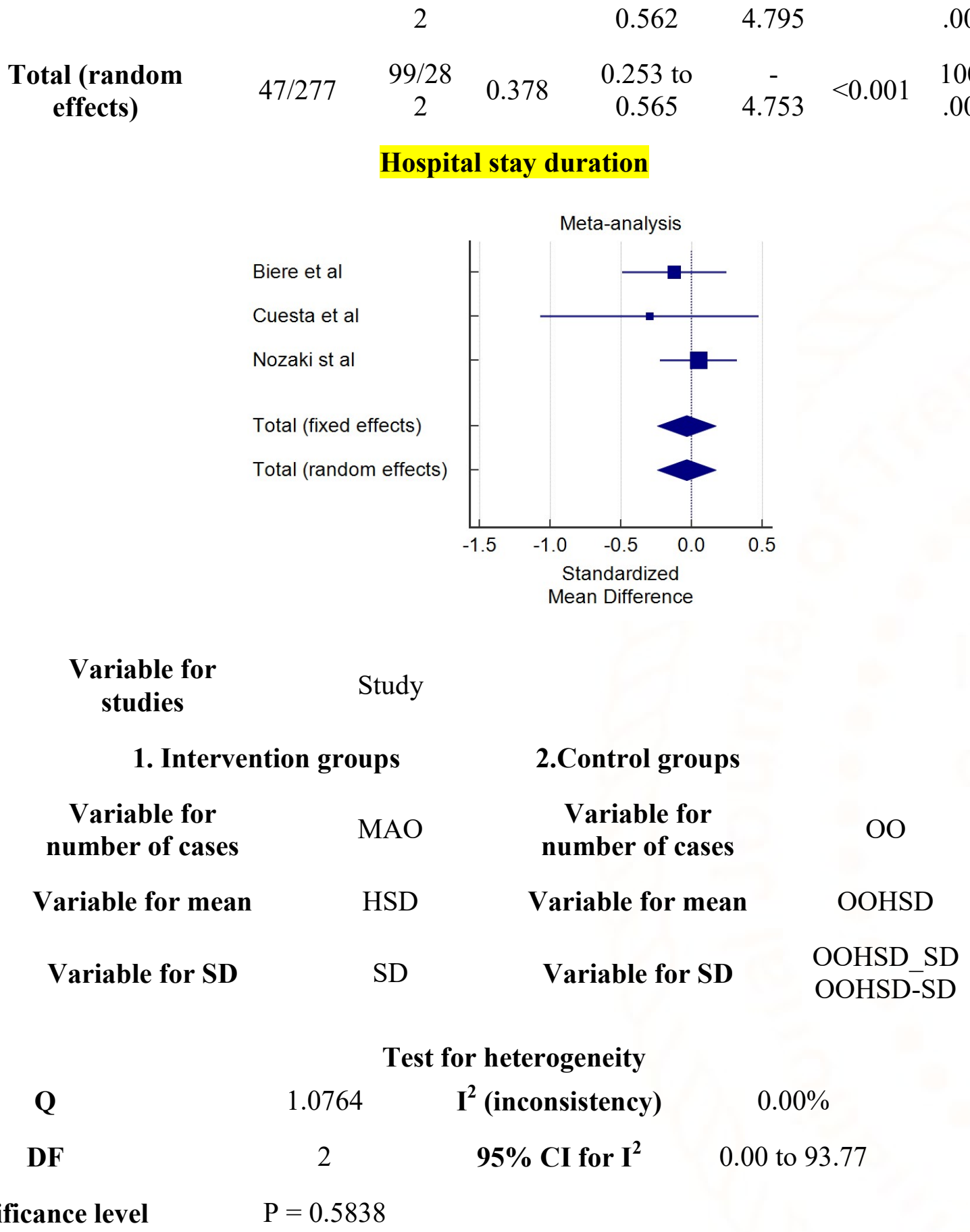

\begin{tabular}{|c|c|c|c|c|c|c|c|c|c|c|}
\hline \multirow[b]{2}{*}{ Study } & \multirow[b]{2}{*}{$\begin{array}{l}\mathbf{N} \\
\mathbf{1}\end{array}$} & \multirow[b]{2}{*}{$\begin{array}{l}\mathbf{N} \\
2\end{array}$} & \multirow[b]{2}{*}{$\begin{array}{c}\text { Tot } \\
\text { al }\end{array}$} & \multirow[b]{2}{*}{ SMD } & \multirow[b]{2}{*}{ SE } & \multirow[b]{2}{*}{$95 \% \mathrm{CI}$} & \multirow[b]{2}{*}{$\mathbf{t}$} & \multirow[b]{2}{*}{$\mathbf{P}$} & \multicolumn{2}{|c|}{$\begin{array}{c}\text { Weight } \\
(\%)\end{array}$} \\
\hline & & & & & & & & & $\begin{array}{c}\text { Fix } \\
\text { ed }\end{array}$ & $\begin{array}{c}\text { Ra } \\
\text { ndo } \\
\text { m }\end{array}$ \\
\hline Biere et al & $\begin{array}{l}5 \\
9\end{array}$ & $\begin{array}{l}5 \\
6\end{array}$ & 115 & $\begin{array}{c}- \\
0.121\end{array}$ & $\begin{array}{l}0.1 \\
85\end{array}$ & $\begin{array}{c}-0.489 \text { to } \\
0.246\end{array}$ & & & $\begin{array}{l}32 . \\
68\end{array}$ & $\begin{array}{l}32 . \\
68\end{array}$ \\
\hline Cuesta et al & $\begin{array}{l}1 \\
4\end{array}$ & $\begin{array}{l}1 \\
3\end{array}$ & 27 & $\begin{array}{c}- \\
0.298\end{array}$ & $\begin{array}{l}0.3 \\
76\end{array}$ & $\begin{array}{c}-1.072 \text { to } \\
0.476\end{array}$ & & & $\begin{array}{c}7.9 \\
7\end{array}$ & $\begin{array}{c}7.9 \\
7\end{array}$ \\
\hline
\end{tabular}




\begin{tabular}{|c|c|c|c|c|c|c|c|c|c|c|}
\hline Nozaki st al & $\begin{array}{l}1 \\
0 \\
1\end{array}$ & $\begin{array}{l}1 \\
0 \\
9\end{array}$ & 210 & $\begin{array}{c}0.048 \\
6\end{array}$ & $\begin{array}{l}0.1 \\
38\end{array}$ & $\begin{array}{c}-0.223 \text { to } \\
0.320\end{array}$ & & & $\begin{array}{l}59 . \\
36\end{array}$ & $\begin{array}{l}59 . \\
36\end{array}$ \\
\hline Total (fixed effects) & $\begin{array}{l}1 \\
7 \\
4\end{array}$ & $\begin{array}{l}1 \\
7 \\
8\end{array}$ & 352 & $\begin{array}{c}- \\
0.034 \\
6\end{array}$ & $\begin{array}{l}0.1 \\
06\end{array}$ & $\begin{array}{c}-0.243 \text { to } \\
0.174\end{array}$ & $\begin{array}{c}- \\
0.32 \\
6\end{array}$ & $\begin{array}{l}0.7 \\
45\end{array}$ & $\begin{array}{l}100 \\
.00\end{array}$ & $\begin{array}{l}100 \\
.00\end{array}$ \\
\hline $\begin{array}{l}\text { Total (random } \\
\text { effects) }\end{array}$ & $\begin{array}{l}1 \\
7 \\
4\end{array}$ & $\begin{array}{l}1 \\
7 \\
8\end{array}$ & 352 & $\begin{array}{c}- \\
0.034 \\
6\end{array}$ & $\begin{array}{l}0.1 \\
06\end{array}$ & $\begin{array}{c}-0.243 \text { to } \\
0.174\end{array}$ & $\begin{array}{c}- \\
0.32 \\
6\end{array}$ & $\begin{array}{l}0.7 \\
45\end{array}$ & $\begin{array}{l}100 \\
.00\end{array}$ & $\begin{array}{l}100 \\
.00\end{array}$ \\
\hline
\end{tabular}

\section{TOTAL DURATION OF SURGERY}

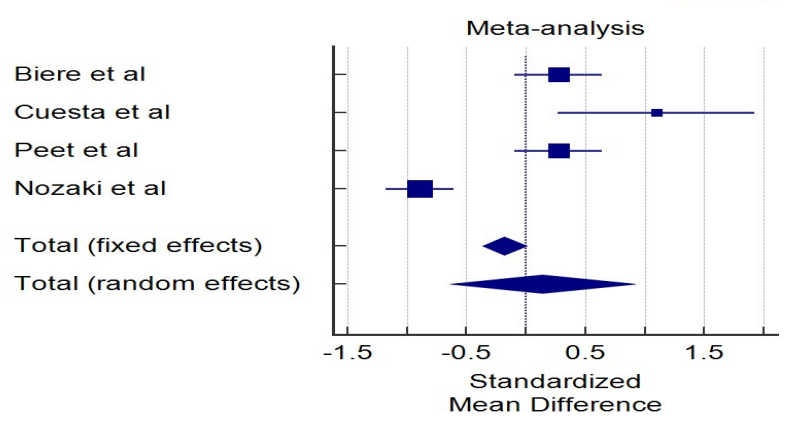

Test for heterogeneity

Q

DF

Significance level

$I^{2}$ (inconsistency)

95\% CI for $\mathbf{I}^{2}$
46.1175

3

$$
\begin{gathered}
\mathrm{P}<0.0001 \\
93.49 \%
\end{gathered}
$$

\begin{tabular}{|c|c|c|c|c|c|c|c|c|c|c|}
\hline \multirow[b]{2}{*}{ Study } & \multirow[b]{2}{*}{ N1 } & \multirow[b]{2}{*}{ N2 } & \multirow[b]{2}{*}{$\begin{array}{l}\text { Tot } \\
\text { al }\end{array}$} & \multirow[b]{2}{*}{ SMD } & \multirow[b]{2}{*}{ SE } & \multirow[b]{2}{*}{$95 \% \mathrm{CI}$} & \multirow[b]{2}{*}{$\mathbf{t}$} & \multirow[b]{2}{*}{$\mathbf{P}$} & \multicolumn{2}{|c|}{ Weight (\%) } \\
\hline & & & & & & & & & $\begin{array}{c}\text { Fix } \\
\text { ed }\end{array}$ & $\begin{array}{c}\text { Rand } \\
\text { om }\end{array}$ \\
\hline Biere et al & 59 & 56 & 115 & 0.272 & 0.186 & -0.0971 to 0.641 & & & $\begin{array}{l}25 . \\
78\end{array}$ & 25.98 \\
\hline Cuesta et al & 14 & 13 & 27 & 1.097 & 0.402 & 0.269 to 1.925 & & & $\begin{array}{c}5.5 \\
2\end{array}$ & 21.45 \\
\hline Peet et al & 59 & 56 & 115 & 0.272 & 0.186 & -0.0971 to 0.641 & & & $\begin{array}{l}25 . \\
78\end{array}$ & 25.98 \\
\hline Nozaki et al & 101 & 109 & 210 & -0.891 & 0.144 & -1.175 to -0.606 & & & $\begin{array}{l}42 . \\
91\end{array}$ & 26.59 \\
\hline Total (fixed & 233 & 234 & 467 & -0.182 & 0.094 & -0.367 to & $\begin{array}{c}- \\
1.92\end{array}$ & 0.0 & 100 & 100.0 \\
\hline
\end{tabular}

86.54 to 96.86 
International Journal of Trend in Scientific Research and Development (IJTSRD) ISSN: 2456-6470

effects)

5

0.00425

$\begin{array}{llll}0 & 55 & .00 & 0\end{array}$

Total

(random

$233 \quad 234 \quad 467$

0.140

0.395

-0.637 to 0.916

$\begin{array}{llll}0.35 & 0.7 & 100 & 100.0\end{array}$ effects)

\begin{tabular}{|c|c|c|c|}
\hline Intervention group & Controls group & OO \\
\hline $\begin{array}{c}\text { Variable for no of } \\
\text { cases }\end{array}$ & MAO & Variable for no of cases & OO-TDS \\
\hline Variable for mean & TDS & Variable for mean & oo-TDS_SD \\
\hline Variable for SD & $\begin{array}{c}\text { TDS- } \\
\text { SD }\end{array}$ & Variable for SD & SD Standard deviation \\
\hline $\begin{array}{c}\text { TDS=Total duration } \\
\text { of }\end{array}$ & $\begin{array}{c}\text { MAO } \\
\text { Surgery }\end{array}$ & $\begin{array}{c}\text { Minimal access } \\
\text { oesophagectomy } \\
\text { Open oesophagectomy }\end{array}$ & \\
\hline
\end{tabular}

\section{BLOOD LOSS}

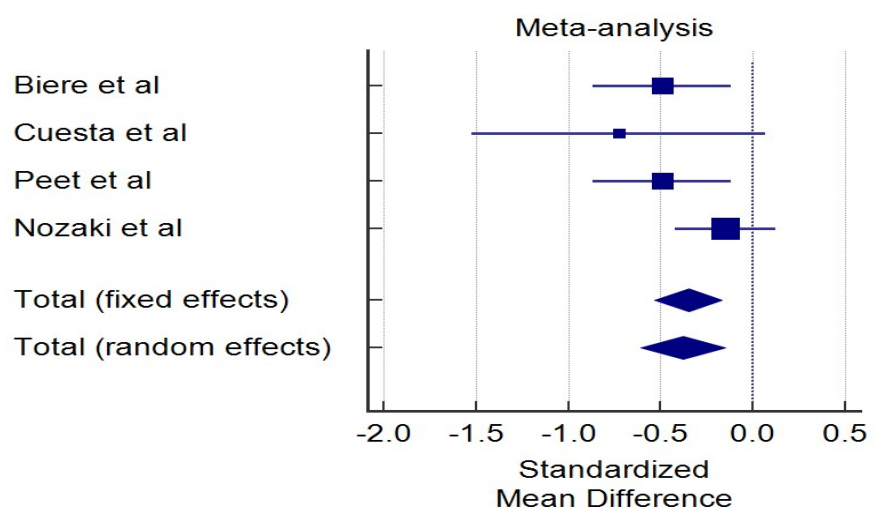

Test for heterogeneity

Q

DF

Significance level

$I^{2}$ (inconsistency)

95\% CI for $\mathrm{I}^{2}$
4.2365

3

$\mathrm{P}=0.2370$

$29.19 \%$

0.00 to 74.01 


\begin{tabular}{|c|c|c|c|c|c|c|c|c|c|}
\hline Peet et al & $\begin{array}{l}5 \\
9\end{array}$ & 56 & 115 & $\begin{array}{c}0.49 \\
2\end{array}$ & $\begin{array}{c}0.18 \\
8\end{array}$ & $\begin{array}{c}-0.865 \text { to }- \\
0.119\end{array}$ & & & 24.38 \\
\hline Nozaki et al & $\begin{array}{l}1 \\
0 \\
1\end{array}$ & 109 & 210 & $\begin{array}{c}- \\
0.14 \\
8\end{array}$ & $\begin{array}{c}0.13 \\
8\end{array}$ & $\begin{array}{c}-0.420 \text { to } \\
0.123\end{array}$ & & & 45.45 \\
\hline $\begin{array}{l}\text { Total (fixed } \\
\text { effects) }\end{array}$ & $\begin{array}{l}2 \\
3 \\
3\end{array}$ & 234 & 467 & $\begin{array}{c}- \\
0.34 \\
9\end{array}$ & $\begin{array}{c}0.09 \\
29\end{array}$ & $\begin{array}{c}-0.532 \text { to }- \\
0.167\end{array}$ & $\begin{array}{c}- \\
3.7 \\
61\end{array}$ & $\begin{array}{c}<0.00 \\
1\end{array}$ & $\begin{array}{c}100.0 \\
0\end{array}$ \\
\hline $\begin{array}{l}\text { Total (random } \\
\text { effects) }\end{array}$ & $\begin{array}{l}2 \\
3 \\
3\end{array}$ & 234 & 467 & $\begin{array}{c}- \\
0.37 \\
8\end{array}$ & $\begin{array}{c}0.11 \\
6\end{array}$ & $\begin{array}{c}-0.607 \text { to }- \\
0.149\end{array}$ & $\begin{array}{c}- \\
3.2 \\
42\end{array}$ & 0.001 & $\begin{array}{c}100.0 \\
0\end{array}$ \\
\hline
\end{tabular}

\begin{tabular}{|c|c|c|c|}
\hline Intervention group & & Controls group & OO \\
\hline $\begin{array}{c}\text { Variable for no of } \\
\text { cases }\end{array}$ & MAO & Variable for no of cases & OO-BL \\
\hline Variable for mean & BL & Variable for mean & 00BLSD \\
\hline Variable for SD & BLSD & Variable for SD & $\begin{array}{c}\text { SD Standard } \\
\text { deviation }\end{array}$ \\
\hline $\mathrm{BL}=$ Blood Loss & $\begin{array}{c}\text { MAO } \\
\text { OO }\end{array}$ & $\begin{array}{c}\text { Minimal access } \\
\text { oesophagectomy } \\
\text { Open oesophagectomy }\end{array}$ & \\
\hline
\end{tabular}

LYMPH NODE RETREIVAL

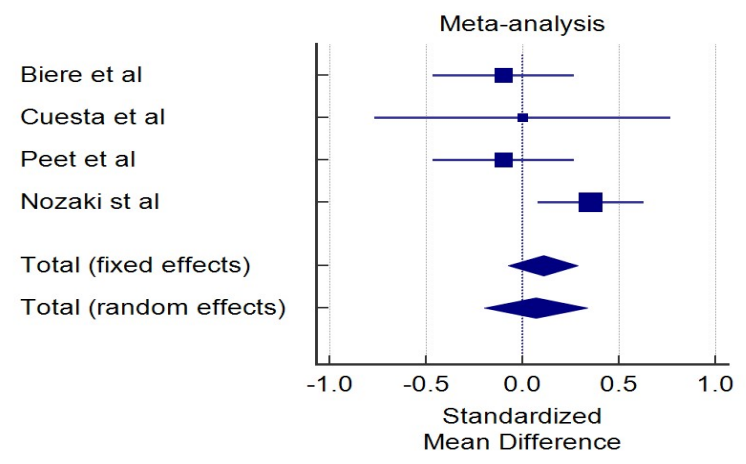

Study

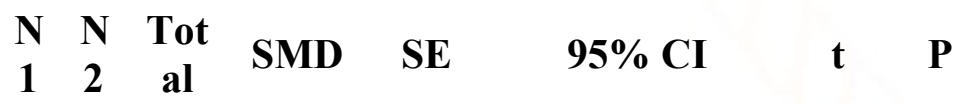

Weight

$(\%)$

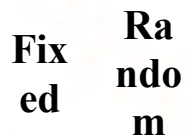
Biere et al
$\begin{array}{ll}5 & 5 \\ 9 & 6\end{array}$
0.098
115
0.18
-0.466 to 0.269
24. 27.
$79 \quad 18$
Cuesta et al
$\begin{array}{ll}1 & 1 \\ 4 & 3\end{array}$
$27 \quad 0.000$
$\begin{array}{cc}0.37 & -0.769 \\ 3 & \text { to } 0.769\end{array}$
6.110.
172
Peet et al
$\begin{array}{cccccc}5 & 5 & 115 & 0.099 & 0.18 & -0.467 \text { to } 0.268 \\ 9 & 6 & & 3 & 5 & \end{array}$
24. 27.
$\begin{array}{ll}79 & 18\end{array}$ 
International Journal of Trend in Scientific Research and Development (IJTSRD) ISSN: 2456-6470

\begin{tabular}{|c|c|c|c|c|c|c|c|c|c|c|}
\hline Nozaki st al & $\begin{array}{l}1 \\
0 \\
1\end{array}$ & $\begin{array}{l}1 \\
0 \\
9\end{array}$ & 210 & 0.353 & $\begin{array}{c}0.13 \\
9\end{array}$ & 0.0800 to 0.627 & & & $\begin{array}{c}44 . \\
31\end{array}$ & $\begin{array}{l}34 . \\
92\end{array}$ \\
\hline Total (fixed effects) & $\begin{array}{l}2 \\
3 \\
3\end{array}$ & $\begin{array}{l}2 \\
3 \\
4\end{array}$ & 467 & 0.108 & $\begin{array}{c}0.09 \\
23\end{array}$ & $\begin{array}{c}-0.0737 \text { to } \\
0.289\end{array}$ & $\begin{array}{l}1.1 \\
66\end{array}$ & $\begin{array}{r}0.2 \\
44\end{array}$ & $\begin{array}{r}100 \\
.00\end{array}$ & $\begin{array}{r}100 \\
.00\end{array}$ \\
\hline $\begin{array}{c}\text { Total (random } \\
\text { effects) }\end{array}$ & $\begin{array}{l}2 \\
3 \\
3\end{array}$ & $\begin{array}{l}2 \\
3 \\
4\end{array}$ & 467 & $\begin{array}{c}0.069 \\
7\end{array}$ & $\begin{array}{c}0.13 \\
6\end{array}$ & -0.198 to 0.338 & $\begin{array}{l}0.5 \\
11\end{array}$ & $\begin{array}{c}0.6 \\
09\end{array}$ & $\begin{array}{r}100 \\
.00\end{array}$ & $\begin{array}{r}100 \\
.00\end{array}$ \\
\hline
\end{tabular}

Test for heterogeneity

Q

DF

\subsection{7}

3

\begin{abstract}
Significance level
$I^{2}$ (inconsistency)

95\% CI for $I^{2}$

Variable for studies Study

1. Intervention groups

Variable for number of cases

Variable for mean

Variable for SD

LNR

SD
\end{abstract}

Methods used for meta-analysis: MedCalc Software is used for meta-analysis in the current review. It utilises Mantel-Haenszel method given by Mantel \& Haenszel in 1959 in order to calculate the odds ratio. The model used by MedCalc during odds ratio based meta-analysis is fixed effects model (Schoonjans, 2016).

Investigation of heterogeneity:The random effects model is used by MedCalc to incorporate heterogeneity. Therefore, the summary odds ratio can be derived(DerSimonian \& Laird, 1986) while performing met a-analysis using MedCalc Software. Cohran's $\mathrm{Q}$ and $\mathrm{I}^{2}$ can be seen as measures of heterogeneity in all the meta-analysis in this review.Cohran's Q detects heterogeneity (Higgins et al., 2003) and $\mathrm{I}^{2}$ represents the percent of observed total variation across all studies which may be

\section{Control groups}

Variable for number of cases

\section{$\mathrm{OO}$}

$\begin{array}{lr}\text { Variable for mean } & \text { OOLNR } \\ \text { Variable for SD } & \text { OOLNR_SD }\end{array}$

because of real heterogeneity rather than chance (Stats Direct Limited, 2000).

Comparisons undertaken: The primary and secondary outcome variables of this review have been compared between the intervention and non-intervention receiving groups of patients. In some studies the intervention is MAO and the control is OO. In the rest the intervention is an experimental factor that is being tested to find if it influences the outcome of MAO versus the control group that does not receive the intervention.

Sensitivity analyses: There has been a dearth of RCTs on MAO. It was very difficult to find the RCTs included in this review and even more difficult to find the ones that meet the inclusion criteria. There are no RCTs whose selection/non-selection for this review is dubious. Also no RCTs have been excluded from this 
review which might have contributed more information except obviously the unpublished ones. Therefore sensitivety analysis is not required.

Sub-group analyses: All the meta analyses in the current review involve some and never all the selected RCTs, because all the selected RCTs are not comparable to each other.

\section{RESULTS}

Characteristics of selected RCTs

The studies included in the current systematic review are RCTs. Only some of the selected RCTs involve a direct comparison of the outcomes of surgery between MAO and OO groups of patients. The data analysis for the other studies which are different from each other is descriptive given below one by one because they cant be compared to each other as they measure different outcomes.

In all the RCTs included in this review, p-values $<0.05$ are regarded significant in statistics. The two groups in all studies are comparable in terms of patient characteristics (shown in the tables $4 \mathrm{a}$ and $4 \mathrm{~b}$ ). All the RCTs included in the review had ethical approval of the local ethical committees /boards. The results of all are valid as checked by critical appraisal tools.

Results of individual studies for outcomes (refer 4-7 to tables for abbreviations)

Ninomiya et al,2010: Statistically significant differences were found in favour of MAO with neutrophil elastase inhibitor versus MAO alone, for the duration of systemic inflammatory response syndrome-SIRS $(p=.009)$, [median arterial oxygen pressure $\{\mathrm{P}\} /\{\mathrm{F}\}$ fraction of inspired oxygen] ratio $(p=.04)$ and interleukin8 levels $(p=.04)$

Biere et al, 2012: The statistically significant differences were found in pulmonary infections $(p=.005)$, short term quality of life and intra-operative blood loss $(\mathrm{p}<.001)$ in favour of MAO versus OO.

Wajed et al, 2012: \{LOGIC TRIAL\} The study showed that perfusion at the anastomotic site did not increase significantly by pre-operative laparoscopic ischaemic conditioning and therefore has no influence on the MAO outcome, anastomotic complications.
Shen et al, 2013: The alveolar lavage fluid interleukin levels (associated with lung inflam mation) 18 hours after MAO were significantly different $\{p<.046$ for all inter leukin levels compared $\}$ between $\mathrm{CV}$ and PV groups. Oxygenation index was significantly lower in $\mathrm{CV}$ than in PV group $(\mathrm{p}=.046)$. Pulmonary complications were significantly higher in $\mathrm{CV}$ group than PV group $(p=.021)$.

Cuesta et al, 2014: Statistically significant differences were found for leucocyte numbers, interleukin8 levels and prolactin levels one week postoperatively in favour of MAO versus $\mathrm{OO}$ with $\mathrm{p}=.004, \mathrm{p}=.047, \mathrm{p}=.049$ respec tively. The lower levels indicate lower inflammatory response and lower immunosuppression. This accounts for fewer infections especially pulmonary infections in MAO group. The finding confirms the previously discussed Ninomiya et al study results that suppression of interleukin 8 by neutrophil elastase inhibitor reduces the postoperative immunosuppression as well as SIRS as a consequence of which pulmonary complications are reduced in the MAO group.

Ito et al, 2005: showed that on day 14 there were no differences in 2 groups in vital capa city and forced expiratory volumes but peak expiratory flow was higher in MAO group than OO group [p<0.05]. Coughing ability was also better after chest physiotherapy. both of which help reduce pulmonary complications

Shen et al, 2014: found that surgery duration was longer in decubitus versus prone posit ion $(p<0.001)$. Blood loss was higher in DP versus PP group ( $p$ $<0.001)$. Ergonomics of surgery were assessed.The surgeons eye blink rate and symptom scale scores were also significantly different between the two groups $(\mathrm{p}<.001)$ proving PP to be better than DP for thoracoscopic MAO.

Nozaki et al, 2014: Two statistically significant findings in this study were that MAO patients had fewer postoperative atelectasis $(11: 24 \mathrm{p}=.041)$ but more risk of re-operation $(10: 2 \mathrm{p}=0.016)$. This study involved very homogenous group of patients all with tumour stage T1bN0M0. Although registered as an RCT numbered JCOG0502 the design of this study was that of a non randomised comparison and caused patient selection bias as well as combina tion bias with the thoracoscopic MAO group more in favour of laparoscopy. 
Takeuchi et al, 2015: The primary outcome weight loss 2 weeks postoperatively, was found significantly lower in the group receiving enteral nutrition than the group who received par enteral nutrition $(p=0.02)$. However it must be noted that although the patient characteris tics and intraoperative factors were comparable, the patients ability to swallow, dehydration etc. influenced the amount of feeding whether enteral or parenteral. This may have influen ced the results of this study.

Peet et al, 2015 : Statistically significant differences were found in the two patient groups for SF36 physical component $(p=.003)$, EORTC C30 global health $(p=.004)$ and EORTC OES18 pain $(p=.001)$ components of quality of life (QOL) questionnares. The limitations include that only $82 \%$ questionnares were completed and trial was not powered for mid term outcomes although it was powered for short-term outcomes. Overall improvement in QOL and rate of late complications in both the groups was found to be equal .

Yamamato et al, 2016: Statistically significant differences in body temperatures were hig her in AA group versus $S$ group at $1 / 2$ hour post administration $(p=.002), 1$ hour after thor acoscopic phase of surgery $(p=.008)$ and 2 hours after leaving the operation theatre $(\mathrm{p}<.001)$; Shivering score was lower in AA versus $S$ group $(p=.049)$. It was noted that statistically sig nificant differences were found in regards to the incidence of infectious complications of surgery $(p=.029)$ betwee AA and $\mathrm{S}$ groups. The univariate analysis revealed that these infec tiouous surgical complications were related to AA administration $(p=.032)$ and blood loss $(p=.025)$. Multivariate analysis showed that AA administration was independently related to reduction in these infectious surgical complications (odds ratio $=.301$ ) and so was blood loss(odds ratio $=.002$ ).

Marriete et al $\{$ the French MIRO trial\} study data is actually from an abstract becuse the full text is not published yet.

Results from Data extraction tables \& Metaanalysis: Data synthesis The meta- analysis of data revealed the following facts:

1) Blood loss during MAO is significantly less than during $\mathrm{OO}(\mathrm{P}<.001)$
2)Post-operative pulmonary infection is significantly lower in MAO versus OO group $(\mathrm{p}<.001)$

3)Postoperative hospital stay duration does not differ significantly between the two groups $(p=0.745)$

4)The Lymph node retreival difference between the two groups is not statistically significant.

5)Total duration of MAO operation is significantly longer than time taken for OO operation $(\mathrm{p}<.0001)$

The statistical and descriptive analysis of other trials revealed the following facts:

6)Laparoscopic ischaemic conditioning does not make any significant changes in the anasto motic complications of MAO.

7)Perioperative amino acid administration reduces hypothermia, inflammatory response and immunosuppression as a consequence of which the pulmonary complications are significan- tly reduced. The infectiouous surgical complications reduce by AA administration $(p=.032)$ and blood loss $(p=.025)$.

8)Selective neutrophil elastase inhibitor administration during MAO leads to reduced lung injury, reduced postoperative hypoxia and reduced immunosuppression all of which lead to reduced incidence of pulmonary complications versus $\mathrm{OO}$.

9)Chest physiotherapy in immediate postoperative period improves coughing ability and peak expiratory flow from day 3 postoperatively in MAO versus OO. As a consequence pulmonary complications get markedly reduced in MAO versus OO.

10)Low tidal volume controlled ventilation (CV) during MAO versus conventional tidal vol ume preserved ventilation (PV) reduces the lung injury of surgery. Oxygenation index is significantly lower in $\mathrm{CV}$ than in PV group $(\mathrm{p}=.046)$. So pulmonary complications get reduced.

11) Statistically significant differences found one week postoperatively in leucocyte numbers, inter leukin8 levels and prolactin levels favour MAO versus $\mathrm{OO}(\mathrm{p}=.004, \mathrm{p}=.047, \mathrm{p}=.049$ respectively).

12) MAO patients have statistically significant fewer postoperative atelectasis versus OO $(\mathrm{p}=.041)$ but more risk of re-operation $(\mathrm{p}=0.016)$. 
13)Weight loss rate 2 weeks postoperatively is significantly lower with enteral nutrition versus parenteral nutrition $(p=0.02)$ after MAO.

14)Statistically significant differences in the quality of life SF36 physical component $(p=.003)$, EORTC C30 global health $(p=.004)$ and EORTC OES18 pain $(p=.001)$ components are found at 6 weeks postoperatively in MAO vs OO.

15)Surgery duration is longer in MAO in DP versus PP group $(p<0.001)$. Blood loss volume was higher in DP versus PP group $(\mathrm{p}<0.001)$.

Discussion- Summary of major findings The current systematic review and sub-group meta-analysis attempts to establish the superiority of MAO outcomes over $\mathrm{OO}$ outcomes and also provides evidence in regards to the ideal MAO technique plus perio-perative interven tions needed for optimising the outcomes of surgery. The aim of the review to establish level 1 evidence based on RCTs in favour of MAO for resectable OCA management has been fulfilled for one primary outcome pu lmonary infections \} and one secondary outcome \{blood loss $\mathrm{du}$ ring surgery $\}$. No statistically significant differences were found for the other primary outco mes - total duration of hospital stay $(p=0.745)$ and lymph node retreival $(\mathrm{p}=1.16)$ between the two groups. The trials studying perioperative interventions proved that significant improve ment in the postoperative outcomes can be brought about by modifying patient position and ventilation techniques, reducing hypothermia and administering factors which reduce systemic inflammation,local lung injury, immunosuppression as a consequence of which the pulmonary complications reduce. This systematic review assessed 12 RCTs totaling 1093 pat ients. None of RCTs provided evidence for reduction in anastomotic leak rate in either group. Previous studies based on case-control/ cohort studies had indicated that anastomotic leak age might be greater in MAO group than $\mathrm{OO}$ but the current review established no statistically significant differences in anastomotic complications between the two groups. Also in one $\mathrm{RCT}$, it has been clearly proven that gastric ischaemic conditioning does not alter anastomosis related complications. The strength of the review is that it is based on RCTs only. So the evidence generated is level 1 evidence which is considered as the best. All the RCTs included had ethical approval and provided valid results. No other systematic review on MAO outcomes based on RCTs alone has been performed till date. This review aims to provide the highest level of evidence that is available till date in favour of MAO outcomes.

An interesting result of the review was that it provided insight into periop erative modificatio ns and how they can influence MAO outcomes. If we combine the results of those trials which involved patients undergoing thoracoscopy, we can hypothesize that prone position thoraco scopy, on low tidal volume ventilation, with administration of neutrophil elastase inhibitor and amino acids can be the ideal thoracic approach for resectable OCA when combined with immediate postoperative chest physiotherapy. All these factors indivisually lead to improved pulmonary outcomes as seen in the RCTs.

By combining the results of the trials the ideal MAO strtegy for resectable OCA patients could be derived. Thus the aim of the review is fulfilled.

Till now MAO has not replaced OO due to lack of high quality evidence and this review aimed to provide that evidence.

\section{Limitations of the review}

The limitations of the study are that

the selected RCTs are small scale studies. -Absence of blinding in some RCTs may reduce the quality of information provided.

Minimal access technique of robotic oesophagectomy and its outcomes have not been discussed as there is no RCT currently that could have been included.

-The

quality of life improvement at 6 weeks was found to be statistically significant in one trial in MAO versus $\mathrm{OO}$ group but this parameter has not been assessed in most of the other trials.

$>$ ICU stay could not be assessed as most of the selected trials provide no information.

$>$ Long term survival and morbidity data is not available through any trial on MAO so far.

$>$ The study by Peet et al included in the metaanalysis actually duplicates the short term data results of Biere et al as it is the same trial followed up for mid term outcomes at year.

\section{Conclusions \& Recommendations.}

Statistically significant differences in terms of blood loss during surgery, post-operative pulmonary infection and total duration of postoperative hospital 
stay, favour MAO versus OO for resectable OCA management.

The ideal MAO strategy for resectable oesophageal cancer may be thoracoscopic oesophagec tomy in prone position with low tidal volume ventilation, plus administration of amino acids with neutrophil elastase inhibitor and immediate postoperative chest physiotherapy with enteral feeding

The abdominal phase of the procedure should ideally be laparoscopy but no conclusion can be made from this study because no RCT has compared laparoscopic phase of MAO with laparotomy or robot-assisted approaches.

Randomised controlled trials involving larger patient numbers may confirm the findings in this review. The seemingly optimal strategy needs to be tested before recommending it as the gold standard of management of resectable OCA. Long term outcomes of MAO also need to be assessed through large scale RCTs .

So, the recommendations of this study are:

$>$ confirm the findings of this review through a systematic review and meta-analysis involving large scale RCTs

$>$ test the optimal MAO strategy found in this trial for resectable OCA management.

$>$ long term follow up studies after MAO procedure be conducted for long term survival, mortality, morbidity and quality of life data.

\section{References /Bibliography}

1) Absi, A., Rice, T.R. and Adelstein, D.J., 2010. Esophageal Cancer. The Cleveland Clinic Foundation. Available at: $<$ http://www.clevelandclinicmeded.com/medicalp ubs/diseasemanagement/hematology-

oncology/esophageal-cancer/> [Accessed 15 September 2016].

2) American Cancer Society, 2016. Treating cancer of the esophagus by stage. Available at: $<$ http://www.cancer.org/cancer/esophaguscancer/d etailedguide/esophagus-cancer-treating-by-stage $>$ [Accessed 15 September 2016].

3) Armijo-Olivo, S., Stiles, C., Hagen, N., Biondo, P. and Cummings, G., 2010. Assessment of study quality for systematic reviews: A comparison of the Cochrane collaboration risk of bias tool and the effective public health practice project quality assessment tool: Methodological research. Journal of evaluation in clinical practice., 18(1), pp.12-8 Available at: $<$ http://www.ncbi.nlm.nih.gov/pubmed/20698919 $>$ [Accessed 24 September 2016].

4) Biere, S., Cuesta, M. and van der Peet, D., 2009. Minimally invasive versus open esophagectomy for cancer: A systematic review and metaanalysis. Minerva Chirurgica, 64(2), pp.121-133. Available at: $<$ http://europepmc.org/abstract/med/19365313> [Accessed 16 September 2016].

5) Biere, S., Maas, K., Bonavina, L., Garcia, J., Berge, van, Rosman, C., Sosef, M., Lange, de, Bonjer, H., Cuesta, M. and der, van, 2011. Traditional invasive vs. Minimally invasive esophagectomy: A multi-center, randomized trial (tIME-trial). BMC surgery., 11 Available at: $<$ http://www.ncbi.nlm.nih.gov/pubmed/21226918 $>$ [Accessed 15 September 2016].

6) Boone, J., Livestro, D., Elias, S., Rinkes, B. and Hillegersberg, van, 2009. International survey on esophageal cancer: Part I surgical techniques. Diseases of the esophagus : official journal of the International Society for Diseases of the Esophagus / I.S.D.E., 22(3), pp.195-202 Available at: $<$ http://www.ncbi.nlm.nih.gov/pubmed/19191856 $>$ [Accessed 18 September 2016].

7) Briez, N., Piessen, G., Bonnetain, F., Brigand, C., Carrere, N., Collet, D., Doddoli, C., Flamein, R., Mabrut, J., Meunier, B., Msika, S., Perniceni, T., Peschaud, F., Prudhomme, M., Triboulet, J. and Mariette, C., 2011. Open versus laparoscopicallyassisted oesophagectomy for cancer: A multicentre randomised controlled phase III trial the MIRO trial. BMC cancer., 11 Available at: $<$ http://www.ncbi.nlm.nih.gov/pubmed/21781337 $>$ [Accessed 14 September 2016].

8) Broussard, B., Evans, J., Wei, B. and Cerfolio, R., 2016. Robotic esophagectomy. The Journal of Visualized Surgery, 2(8) Available at: $<$ http://jovs.amegroups.com/article/view/11263/11 842> [Accessed 15 September 2016].

9) Burdall, O.C., Fullick, J., Boddy, A.P. and Blazeby, jane, 2016. Improved survival following minimally invasive Oesophagectomy compared to open surgery - SAGES abstract archives. SAGES. Available at: $<$ http://www.sages.org/meetings/annualmeeting/abstracts-archive/improved-survival- 
International Journal of Trend in Scientific Research and Development (IJTSRD) ISSN: 2456-6470

following-minimally-invasive-oesophagectomycompared-to-open-surgery/> [Accessed 17 September 2016].

10) Cancer Research UK, 2016. About oesophageal cancer. Available at: $<$ http://www.cancerresearchuk.org/aboutcancer/oesophageal-cancer/about> [Accessed 15 September 2016].

11) Cancer today IARC, 2012. CANCER FACT SHEETS: OESOPHAGEAL CANCER. [WHO IARC] France: Globocan. Available at: $<$ http://gco.iarc.fr/today/fact-sheetscancers? cancer $=4 \&$ type $=0 \&$ sex $=0>$ Accessed 15 September 2016].

12) CASP, UK, 2013. Critical appraisal skills Programme (CASP). Critical Appraisal Skills Programme (CASP). Available at: $<$ http://www.casp-uk.net/casp-tools-checklists> [Accessed 23 September 2016].

13) Chai, J. and Jamal, M.M., 2012. Esophageal malignancy: A growing concern18(45). Available at:

$<$ http://www.ncbi.nlm.nih.gov/pmc/articles/PMC3 516225/> [Accessed 18 September 2016].

14) Collard, J.-M., Lengele, B., Kestens, P.-J. and Otte, J.B., 1996. En bloc and standard esophagectomies by thoracoscopy. [Volume 61, Issue 2, Pages 769-770] The Annals of Thoracic Surgery. Available at: $<$ http://www.annalsthoracicsurgery.org/article/000 3-4975(95)00951-5/abstract> [Accessed 18 September 2016].

15) Cuschieri, A., Shimi, S. and Banting, S., 1992. Endoscopic oesophagectomy through a right thoracoscopic approach. Journal of the Royal College of Surgeons of Edinburgh., 37(1), pp.7-11 Available at: $<$ http://www.ncbi.nlm.nih.gov/pubmed/1573620> [Accessed 18 September 2016].

16) Dantoc, M., Cox and Eslick, G., 2012a. Evidence to support the use of minimally invasive esophagectomy for esophageal cancer: A metaanalysis. Archives of surgery (Chicago, Ill. : 1960)., 147(8), pp.768-76 Available at: $<$ http://www.ncbi.nlm.nih.gov/pubmed/22911078 ?dopt=Abstract $>$ [Accessed 16 September 2016].

17) Dantoc, M.M., Cox, M.R. and Eslick, G.D., 2011. Does minimally invasive Esophagectomy (MIE) provide for comparable Oncologic outcomes to open techniques? A systematic review. Journal of Gastrointestinal Surgery, 16(3), pp.486-494.
18) Dantoc, M.M., Cox, M.R. and Eslick, G.D., 2012b. The first randomised controlled trial on minimally invasive esophagectomy (MIE) and the ongoing quest for greater evidence4(5). Available at:

$<$ http://www.ncbi.nlm.nih.gov/pmc/articles/PMC3 461080/> [Accessed 17 September 2016].

19) Enzinger, P.C. and Mayer, R.J., 2003. Esophageal cancer. New England Journal of Medicine, 349(23), pp.2241-2252.

20) First and Adenis, A., 2016. 140102-158. Available at:

$<$ http://meetinglibrary.asco.org/content/140102158> [Accessed 14 September 2016].

21) Fujita, T., Okada, N., Kanamori, J., Sato, T., Mayanagi, S., Torigoe, K., Oshita, A., Yamamoto, H. and Daiko, H., 2016. Thermogenesis induced by amino acid administration prevents intraoperative hypothermia and reduces postoperative infectious complications after thoracoscopic esophagectomy. Diseases of the esophagus: official journal of the International Society for Diseases of the Esophagus / I.S.D.E., Available at: $<$ http://www.ncbi.nlm.nih.gov/pubmed/27003457 $>$ [Accessed 14 September 2016].

22) Gemmill, E.H. and McCulloch, P., 2007. Systematic review of minimally invasive resection for gastro-oesophageal cancer. British Journal of Surgery, 94(12), pp.1461-1467.

23) Gockel, I., Exner, C. and Junginger, T., 2005. Morbidity and mortality after esophagectomy for esophageal carcinoma: A risk analysis. World Journal of Surgical Oncology, 3(1), p.37.

24) Gurusamy, K., Pallari, E., Midya, S. and Mughal, M., 2016. Laparoscopic versus open transhiatal oesophagectomy for oesophageal cancer. The Cochrane database of systematic reviews., 3 Available at: $<$ http://www.ncbi.nlm.nih.gov/pubmed/27030301 $>$ [Accessed 16 September 2016].

25) Haughom, J. and Advisor, S., 2015. 5 reasons the practice of evidence-based medicine is a hot topic. Health Catalyst. Available at: $<$ http://www.healthcatalyst.com/5-reasonspractice-evidence-based-medicine-is-hot-topic $>$ [Accessed 16 September 2016].

26) Kawahara, Y., Ninomiya, I., Fujimura, T., Funaki, H., Nakagawara, H., Takamura, H., Oyama, K., Tajima, H., Fushida, S., Inaba, H. and Kayahara, M., 2009. Prospective randomized controlled study on the effects of perioperative 
administration of a neutrophil elastase inhibitor to patients undergoing video-assisted thoracoscopic surgery for thoracic esophageal cancer. Diseases of the esophagus: official journal of the International Society for Diseases of the Esophagus / I.S.D.E., 23(4), pp.329-39 Available at:

$<$ http://www.ncbi.nlm.nih.gov/pubmed/19788440 $>$ [Accessed 14 September 2016].

27) Kim, T., Hochwald, S.N., Sarosi, G.A., Caban, A.M., Rossidis, G., Ben-David, K. and Corporation, H.P., 2012. Review of minimally invasive Esophagectomy and current controversies. Gastroenterology Research and Practice, 2012. Available at: $<$ https://www.hindawi.com/journals/grp/2012/683 213/> [Accessed 16 September 2016].

28) Luketich, J.D., Alvelo-Rivera, M., Buenaventura, P.O., Christie, N.A., McCaughan, J.S., Litle, V.R., Schauer, P.R., Close, J.M. and Fernando, H.C., 2003. Minimally invasive Esophagectomy: Outcomes in 222 patients: Annals of surgery. LWW. Available

at: $<$ http://journals.lww.com/annalsofsurgery/Abstrac t/2003/10000/Minimally_Invasive_Esophagectom y_Outcomes_in_222.4.aspx $>$ [Accessed 17 September 2016].

29) Luketich, J., Pennathur, A., Franchetti, Y., Catalano, P., Swanson, S., Sugarbaker, D., Hoyos, D., Maddaus, M., Nguyen, N., Benson, A. and Fernando, H., 2015. Minimally invasive esophagectomy: Results of a prospective phase II multicenter trial-the eastern cooperative oncology group (E2202) study. Annals of surgery., 261(4), pp.702-7 Available at: $<$ http://www.ncbi.nlm.nih.gov/pubmed/25575253 $>$ [Accessed 14 September 2016].

30) Melhado, R.E., Alderson, D. and Tucker, O., 2010. The changing face of Esophageal cancer. Cancers, 2(3), pp.1379-1404. Available at: $<$ http://www.mdpi.com/2072-6694/2/3/1379/htm> [Accessed 15 September 2016].

31) Merkow, R.P., Bilimoria, K.Y., Chow, W.B., Merkow, J.S., Weyant, M.J., Ko, C.Y. and Bentrem, D.J., 2012. Variation in lymph Node examination after Esophagectomy for cancer in the United States. Archives of Surgery, 147(6), pp.505-511. Available

at: $<$ http://archsurg.jamanetwork.com/article.aspx?art icleid $=1150116>$ [Accessed 25 September 2016].

32) Nagpal, K., Ahmed, K., Vats, A., Yakoub, D., James, D., Ashrafian, H., Darzi, A., Moorthy, K. and Athanasiou, T., 2010. Is minimally invasive surgery beneficial in the management of esophageal cancer? A meta-analysis. Surgical endoscopy., 24(7), pp.1621-9 Available at: $<$ http://www.ncbi.nlm.nih.gov/pubmed/20108155 ?dopt $=$ Abstract $>$ [Accessed 16 September 2016].

33) Nakatsuchi, T., Otani, M., Osugi, H., Ito, Y. and Koike, T., 2005. The necessity of chest physical therapy for thoracoscopic oesophagectomy. The Journal of international medical research., 33(4), pp.434-41 Available

at: $<$ http://www.ncbi.nlm.nih.gov/pubmed/16104447 $>$ [Accessed 14 September 2016].

34) Nguyen, N.T., Hinojosa, M.W., Smith, B.R., Chang, K.J., Gray, J. and Hoyt, D., 2008. Minimally invasive esophagectomy lessons learned from 104 operations. Annals of Surgery, 248(6), p.10811091 Available at: $<$ http://www.ncbi.nlm.nih.gov/pubmed/19092354 $>$ [Accessed 16 September 2016].

35) Nozaki, I., Kato, K., Igaki, H., Ito, Y., Daiko, H., Yano, M., Udagawa, H., Mizusawa, J., Katayama, H., Nakamura, K. and Kitagawa, Y., 2015. Evaluation of safety profile of thoracoscopic esophagectomy for T1bN0M0 cancer using data from JCOG0502: A prospective multicenter study. Surgical endoscopy., 29(12), pp.3519-26 Available at: $<$ http://www.ncbi.nlm.nih.gov/pubmed/25676203 $>$ [Accessed 14 September 2016].

36) Oesophageal cancer incidence statistics, 2016. Cancer Research UK. Available at: $<$ http://www.cancerresearchuk.org/healthprofessional/cancer-statistics/statistics-by-cancertype/oesophageal-cancer/incidence\#headingTwo $>$ [Accessed 15 September 2016].

37) Park, D.P., Welch, C.A., Harrison, D.A., Palser, T.R., Cromwell, D.A., Gao, F., Alderson, D., Rowan, K.M. and Perkins, G.D., 2009. Outcomes following oesophagectomy in patients with oesophageal cancer: A secondary analysis of the ICNARC case mix Programme database. Critical Care, 13(Suppl 2), p.S1.

38) Pennathur, A., Gibson, M.K., Jobe, B.A. and Luketich, J.D., $2016 . \quad$ Oesophageal carcinoma381(9864), pp.400-412. Available at: $<$ http:/www.sciencedirect.com/science/article/pii/ S0140673612606436> [Accessed 17 September 2016].

39) Peters, C.J. and Fitzgerald, R.C., 2007. Systematic Review: The Application of Molecular Pathogenesis to Prevention and Treatment of 
International Journal of Trend in Scientific Research and Development (IJTSRD) ISSN: 2456-6470

Oesophageal Adenocarcinoma. [Aliment Pharmacol Ther.] Available at: $<$ http://www.medscape.com/viewarticle/557839> [Accessed 15 September 2016].

40) Relevo, R., 2012. Effective search strategies for systematic reviews of medical tests. [Rockville] NCBI Bookshelf. Available at: $<$ http://www.ncbi.nlm.nih.gov/books/NBK98242/ $>$ [Accessed 18 September 2016].

41) Savides, T.J., 2016. Endoscopic Ultrasound. [GastroHep.com] Wiley-Blackwell. Available at: $<$ http://www.gastrohep.com/ebooks/thumbnails.as p?book=1405120819\&id=3> [Accessed 15 September 2016].

42) Schardt, C., Adams, M.B., Owens, T., Keitz, S. and Fontelo, P., 2007. Utilization of the PICO framework to improve searching PubMed for clinical questions. BMC Medical Informatics and Decision Making, 7(1), p.16.

43) Scheepers, J.J.G., Cuesta, M.A., Donald L van der Peet and Alexander A F A Veenhof, 2007. Thoracoscopic resection for esophageal cancer: A review of literature3(4). Available at: $<$ http://www.ncbi.nlm.nih.gov/pmc/articles/PMC2 749198/> [Accessed 16 September 2016].

44) Schoonjans, F., 2016. MedCalc $\square$ statistics for biomedical research software manual.. Available at:

$<$ https://www.medcalc.org/download/medcalcman ual.pdf $>$ [Accessed 27 September 2016].

45) Senthilnathan, P., Rajan, P.S., Vaithiswaran, S, R., P, P., C, P. and PMC, E., 2010. Minimally invasive oesophagectomy for carcinoma oesophagus--approaches and options in a high volume tertiary centre.Journal of the Indian Medical Association, 108(10), pp.642-644. Available $<$ http://europepmc.org/abstract/med/21510545> [Accessed 16 September 2016].

46) Sgourakis, G., Gockel, I., Radtke, A., Musholt, T.J., Timm, S., Rink, A., Tsiamis, A., Karaliotas, C. and Lang, H., 2010. Minimally invasive versus open Esophagectomy: Meta-Analysis of outcomes. Digestive Diseases and Sciences, 55(11), pp.3031-3040.

47) Shen, Y., Feng, M., Tan, L., Wang, H., Li, J. and $\mathrm{Xi}$, Y., 2014. Thoracoscopic esophagectomy in prone versus decubitus position: Ergonomic evaluation from a randomized and controlled study. The Annals of thoracic surgery., 98(3), pp.1072-8 Available at:
$<$ http://www.ncbi.nlm.nih.gov/pubmed/25038015 $>$ [Accessed 14 September 2016].

48) Shen, Y., Zhong, M., Wu, W., Wang, H., Feng, M. and Tan, L., 2013. The impact of tidal volume on pulmonary complications following minimally invasive esophagectomy: A randomized and controlled study. The Journal of thoracic and cardiovascular surgery., 146(5), pp.1267-73 Available at: $<$ http://www.ncbi.nlm.nih.gov/pubmed/23993028 $>$ [Accessed 14 September 2016].

49) Smithers, B.M., Gotley, D.C., Martin, I. and Thomas, J.M., 2007. Comparison of the outcomes between open and minimally invasive Esophagectomy245(2) Available at: $<$ http://www.ncbi.nlm.nih.gov/pmc/articles/PMC1 876975/> [Accessed 15 September 2016].

50) StatsDirect Limited, 2000. Heterogeneity in Metaanalysis $(Q, \quad i$-square $)$ Available at: $<$ http://www.statsdirect.com/help/meta_analysis/h eterogeneity.htm $>$ [Accessed 27 September 2016].

51) Taylor, C., 2016. Range rule for standard deviation how to estimate the standard deviation. About.com. Available at: $<$ http://statistics.about.com/od/Descriptive-

Statistics/a/Range-Rule-For-Standard-

Deviation.htm $>$ [Accessed 27 September 2016].

52) Titcomb, D.R., Burdall, O.C., Fullick, J., P Boddy, A., Blazeby, J., Krysztopik, R., Streets, C., Hollowood, A. and Barham, C.P., 2016. Improved survival following minimally invasive Oesophagectomy compared to open surgery SAGES abstract archives. SAGES. Available at: $<$ http://www.sages.org/meetings/annualmeeting/abstracts-archive/improved-survivalfollowing-minimally-invasive-oesophagectomycompared-to-open-surgery/> [Accessed 15 September 2016].

53) Verghese, R., EJ, H., J, B., R, V.H. and PMC, E., 2009. Minimally invasive surgery compared to open procedures in esophagectomy for cancer: A systematic review of the literature. Minerva Chirurgica, 64(2), pp.135-146. Available at: $<$ http://europepmc.org/abstract/med/19365314>

[Accessed 16 September 2016].

54) WCRFI, 2015. Cancer facts \& figures Worldwide data. World cancer research fund international. Available at: $<$ http://www.wcrf.org/int/cancer-factsfigures/worldwide-data $>$ [Accessed 15 September 2016]. 
55) Yamamoto, M., Weber, J.M., Karl, R.C. and Meredith, K.L., 2013. Minimally invasive surgery for Esophageal cancer: Review of the literature and institutional experience20(2) Available at: $<$ https://moffitt.org/File\%20Library/Main\%20Nav /Research\%20and $\% 20$ Clinical\%20Trials/Cancer\% 20Control\%20Journal/v20n2/130.pdf> [Accessed 15 September 2016].

\section{Citations, Quotes \& Annotations}

Absi, A., Rice, T.R. and Adelstein, D.J., 2010. Esophageal Cancer. The Cleveland Clinic Foundation. Available at: $<$ http://www.clevelandclinicmeded.com/medicalpubs/ diseasemanagement/hematologyoncology/esophageal-cancer/> [Accessed 15

September 2016].

(Absi, Rice and Adelstein, 2010)

American Cancer Society, 2016. Treating cancer of the esophagus by stage. Available at: $<$ http://www.cancer.org/cancer/esophaguscancer/detai ledguide/esophagus-cancer-treating-by-stage $>$ [Accessed 15 September 2016].

\section{(American Cancer Society, 2016)}

Armijo-Olivo, S., Stiles, C., Hagen, N., Biondo, P. and Cummings, G., 2010. Assessment of study quality for systematic reviews: A comparison of the Cochrane collaboration risk of bias tool and the effective public health practice project quality assessment tool: Methodological research. Journal of evaluation in clinical practice., 18(1), pp.12-8 Available at: $<$ http://www.ncbi.nlm.nih.gov/pubmed/20698919> [Accessed 24 September 2016].

\section{(Armijo-Olivo et al., 2010)}

Biere, S., Cuesta, M. and van der Peet, D., 2009. Minimally invasive versus open esophagectomy for cancer: A systematic review and meta-analysis. Minerva Chirurgica, 64(2), pp.121-133. Available at: $<$ http://europepmc.org/abstract/med/19365313> [Accessed 16 September 2016].

(Biere, Cuesta and van der Peet, 2009)

Biere, S., Maas, K., Bonavina, L., Garcia, J., Berge, van, Rosman, C., Sosef, M., Lange, de, Bonjer, H.,
Cuesta, M. and der, van, 2011. Traditional invasive vs. Minimally invasive esophagectomy: A multicenter, randomized trial (tIME-trial). BMC surgery., 11 Available at: $<$ http://www.ncbi.nlm.nih.gov/pubmed/21226918> [Accessed 15 September 2016].

(Biere et al., 2011)

Boone, J., Livestro, D., Elias, S., Rinkes, B. and Hillegersberg, van, 2009. International survey on esophageal cancer: Part I surgical techniques. Diseases of the esophagus: official journal of the International Society for Diseases of the Esophagus / I.S.D.E., 22(3), pp.195-202 Available at: $<$ http://www.ncbi.nlm.nih.gov/pubmed/19191856>

[Accessed 18 September 2016].

(Boone et al., 2009)

Briez, N., Piessen, G., Bonnetain, F., Brigand, C., Carrere, N., Collet, D., Doddoli, C., Flamein, R., Mabrut, J., Meunier, B., Msika, S., Perniceni, T., Peschaud, F., Prudhomme, M., Triboulet, J. and Mariette, C., 2011. Open versus laparoscopicallyassisted oesophagectomy for cancer: A multicentre randomised controlled phase III trial - the MIRO trial. BMC cancer., 11 Available at: $<$ http://www.ncbi.nlm.nih.gov/pubmed/21781337>

[Accessed 14 September 2016].

(Briez et al., 2011)

Broussard, B., Evans, J., Wei, B. and Cerfolio, R., 2016. Robotic esophagectomy. The Journal of Visualized Surgery, 2(8) Available at: $<$ http://jovs.amegroups.com/article/view/11263/11842 $>$ [Accessed 15 September 2016].

(Broussard et al., 2016)

Burdall, O.C., Fullick, J., Boddy, A.P. and Blazeby, jane, 2016. Improved survival following minimally invasive Oesophagectomy compared to open surgery SAGES abstract archives. SAGES. Available at: $<$ http://www.sages.org/meetings/annualmeeting/abstracts-archive/improved-survivalfollowing-minimally-invasive-oesophagectomycompared-to-open-surgery/> [Accessed 17 September 2016].

(Burdall et al., 2016) 
Cancer Research UK, 2016. About oesophageal cancer. Available $<$ http://www.cancerresearchuk.org/aboutcancer/oesophageal-cancer/about $>$ [Accessed 15 September 2016].

(Cancer Research UK, 2016)

Cancer today IARC, 2012. CANCER FACT SHEETS: OESOPHAGEAL CANCER. [WHO IARC] France: Globocan. Available at: $<\mathrm{http}: / /$ gco.iarc.fr/today/factsheets-cancers? cancer $=4 \&$ type $=0 \&$ sex $=0>$ [Accessed 15 September 2016].

(Cancer today IARC, 2012)

CASP, UK, 2013. Critical appraisal skills Programme (CASP). Critical Appraisal Skills Programme (CASP). Available at: $<$ http://www.caspuk.net/casp-tools-checklists $>$ [Accessed 23 September 2016].

(CASP, UK, 2013)

Chai, J. and Jamal, M.M., 2012. Esophageal malignancy: A growing concern18(45). Available at: $<$ http://www.ncbi.nlm.nih.gov/pmc/articles/PMC3516 225/> [Accessed 18 September 2016].

(Chai and Jamal, 2012)

Collard, J.-M., Lengele, B., Kestens, P.-J. and Otte, J.B., 1996. En bloc and standard esophagectomies by thoracoscopy. [Volume 61, Issue 2, Pages 769-770] The Annals of Thoracic Surgery. Available at: $<$ http://www.annalsthoracicsurgery.org/article/00034975(95)00951-5/abstract> [Accessed 18 September 2016].

(Collard et al., 1996)

Cuschieri, A., Shimi, S. and Banting, S., 1992. Endoscopic oesophagectomy through a right thoracoscopic approach. Journal of the Royal College of Surgeons of Edinburgh., 37(1), pp.7-11 Available at: <http://www.ncbi.nlm.nih.gov/pubmed/1573620> [Accessed 18 September 2016].

(Cuschieri, Shimi and Banting, 1992)

Dantoc, M., Cox and Eslick, G., 2012a. Evidence to support the use of minimally invasive esophagectomy for esophageal cancer: A meta-analysis. Archives of surgery (Chicago, Ill.: 1960)., 147(8), pp.768-76 Available

at:

$<$ http://www.ncbi.nlm.nih.gov/pubmed/22911078?do

$\mathrm{pt}=$ Abstract $>$ [Accessed 16 September 2016].

(Dantoc, Cox and Eslick, 2012a)

Dantoc, M.M., Cox, M.R. and Eslick, G.D., 2011. Does minimally invasive Esophagectomy (MIE) provide for comparable Oncologic outcomes to open techniques? A systematic review. Journal of Gastrointestinal Surgery, 16(3), pp.486-494.

(Dantoc, Cox and Eslick, 2011)

Dantoc, M.M., Cox, M.R. and Eslick, G.D., 2012b. The first randomised controlled trial on minimally invasive esophagectomy (MIE) and the ongoing quest for greater evidence4(5). Available at: $<$ http://www.ncbi.nlm.nih.gov/pmc/articles/PMC3461 080/> [Accessed 17 September 2016].

(Dantoc, Cox and Eslick, 2012b)

Enzinger, P.C. and Mayer, R.J., 2003. Esophageal cancer. New England Journal of Medicine, 349(23), pp.2241-2252.

(Enzinger and Mayer, 2003)

First and Adenis, A., 2016. 140102-158. Available at: $<$ http://meetinglibrary.asco.org/content/140102-158> [Accessed 14 September 2016].

(First and Adenis, 2016)

Fujita, T., Okada, N., Kanamori, J., Sato, T., Mayanagi, S., Torigoe, K., Oshita, A., Yamamoto, H. and Daiko, H., 2016. Thermogenesis induced by amino acid administration prevents intraoperative hypothermia and reduces postoperative infectious complications after thoracoscopic esophagectomy. Diseases of the esophagus: official journal of the International Society for Diseases of the Esophagus / I.S.D.E., Available at: $<$ http://www.ncbi.nlm.nih.gov/pubmed/27003457> [Accessed 14 September 2016].

(Fujita et al., 2016) 
Gemmill, E.H. and McCulloch, P., 2007. Systematic review of minimally invasive resection for gastrooesophageal cancer. British Journal of Surgery, 94(12), pp.1461-1467.

(Gemmill and McCulloch, 2007)

Gockel, I., Exner, C. and Junginger, T., 2005. Morbidity and mortality after esophagectomy for esophageal carcinoma: A risk analysis. World Journal of Surgical Oncology, 3(1), p.37.

(Gockel, Exner and Junginger, 2005)

Gurusamy, K., Pallari, E., Midya, S. and Mughal, M., 2016. Laparoscopic versus open transhiatal oesophagectomy for oesophageal cancer. The Cochrane database of systematic reviews., 3 Available at: $<$ http://www.ncbi.nlm.nih.gov/pubmed/27030301> [Accessed 16 September 2016].

(Gurusamy et al., 2016)

Haughom, J. and Advisor, S., 2015. 5 reasons the practice of evidence-based medicine is a hot topic. Health Catalyst. Available at: $<$ http://www.healthcatalyst.com/5-reasons-practiceevidence-based-medicine-is-hot-topic $>$ [Accessed 16 September 2016].

(Haughom and Advisor, 2015)

Kawahara, Y., Ninomiya, I., Fujimura, T., Funaki, H., Nakagawara, H., Takamura, H., Oyama, K., Tajima, H., Fushida, S., Inaba, H. and Kayahara, M., 2009. Prospective randomized controlled study on the effects of perioperative administration of a neutrophil elastase inhibitor to patients undergoing videoassisted thoracoscopic surgery for thoracic esophageal cancer. Diseases of the esophagus : official journal of the International Society for Diseases of the Esophagus / I.S.D.E., 23(4), pp.329-39 Available at: $<$ http://www.ncbi.nlm.nih.gov/pubmed/19788440> [Accessed 14 September 2016].

(Kawahara et al., 2009)

Kim, T., Hochwald, S.N., Sarosi, G.A., Caban, A.M., Rossidis, G., Ben-David, K. and Corporation, H.P., 2012. Review of minimally invasive Esophagectomy and current controversies. Gastroenterology Research and Practice, 2012. Available at: $<$ https://www.hindawi.com/journals/grp/2012/683213 $>$ [Accessed 16 September 2016].

(Kim et al., 2012)

Luketich, J.D., Alvelo-Rivera, M., Buenaventura, P.O., Christie, N.A., McCaughan, J.S., Litle, V.R., Schauer, P.R., Close, J.M. and Fernando, H.C., 2003. Minimally invasive Esophagectomy: Outcomes in 222 patients: Annals of surgery. LWW. Available at: $<$ http://journals.lww.com/annalsofsurgery/Abstract/20 03/10000/Minimally_Invasive_Esophagectomy_Out comes_in_222.4.aspx $>$ [Accessed 17 September 2016].

(Luketich et al., 2003)

Luketich, J., Pennathur, A., Franchetti, Y., Catalano, P., Swanson, S., Sugarbaker, D., Hoyos, D., Maddaus, M., Nguyen, N., Benson, A. and Fernando, H., 2015. Minimally invasive esophagectomy: Results of a prospective phase II multicenter trial-the eastern cooperative oncology group (E2202) study. Annals of surgery., 261(4), pp.702-7 Available at: $<$ http://www.ncbi.nlm.nih.gov/pubmed/25575253> [Accessed 14 September 2016].

(Luketich et al., 2015)

Melhado, R.E., Alderson, D. and Tucker, O., 2010. The changing face of Esophageal cancer. Cancers, 2(3), pp.1379-1404. Available at: $<$ http://www.mdpi.com/2072-6694/2/3/1379/htm> [Accessed 15 September 2016].

(Melhado, Alderson and Tucker, 2010)

Merkow, R.P., Bilimoria, K.Y., Chow, W.B., Merkow, J.S., Weyant, M.J., Ko, C.Y. and Bentrem, D.J., 2012. Variation in lymph Node examination after Esophagectomy for cancer in the United States. Archives of Surgery, 147(6), pp.505-511. Available at:

$<$ http://archsurg.jamanetwork.com/article.aspx?article $\mathrm{id}=1150116>$ [Accessed 25 September 2016].

(Merkow et al., 2012)

Nagpal, K., Ahmed, K., Vats, A., Yakoub, D., James, D., Ashrafian, H., Darzi, A., Moorthy, K. and Athanasiou, T., 2010. Is minimally invasive surgery 
beneficial in the management of esophageal cancer? A meta-analysis. Surgical endoscopy., 24(7), pp.1621-9 Available at: $<$ http://www.ncbi.nlm.nih.gov/pubmed/20108155?do $\mathrm{pt}=$ Abstract $>$ [Accessed 16 September 2016].

(Nagpal et al., 2010)

Nakatsuchi, T., Otani, M., Osugi, H., Ito, Y. and Koike, T., 2005. The necessity of chest physical therapy for thoracoscopic oesophagectomy. The Journal of international medical research., 33(4), pp.434-41 Available at: $<$ http://www.ncbi.nlm.nih.gov/pubmed/16104447> [Accessed 14 September 2016].

(Nakatsuchi et al., 2005)

Nguyen, N.T., Hinojosa, M.W., Smith, B.R., Chang, K.J., Gray, J. and Hoyt, D., 2008. Minimally invasive esophagectomy lessons learned from 104 operations. Annals of Surgery, 248(6), p.10811091 Available at: $<$ http://www.ncbi.nlm.nih.gov/pubmed/19092354> [Accessed 16 September 2016].

(Nguyen et al., 2008)

Nozaki, I., Kato, K., Igaki, H., Ito, Y., Daiko, H., Yano, M., Udagawa, H., Mizusawa, J., Katayama, H., Nakamura, K. and Kitagawa, Y., 2015. Evaluation of safety profile of thoracoscopic esophagectomy for T1bN0M0 cancer using data from JCOG0502: A prospective multicenter study. Surgical endoscopy., 29(12), pp.3519-26 Available at: $<$ http://www.ncbi.nlm.nih.gov/pubmed/25676203> [Accessed 14 September 2016].

(Nozaki et al., 2015)

Oesophageal cancer incidence statistics, 2016. Cancer Research UK. Available at: $<$ http://www.cancerresearchuk.org/healthprofessional/cancer-statistics/statistics-by-cancertype/oesophageal-cancer/incidence\#heading-Two> [Accessed 15 September 2016].

(Oesophageal cancer incidence statistics, 2016)

Park, D.P., Welch, C.A., Harrison, D.A., Palser, T.R., Cromwell, D.A., Gao, F., Alderson, D., Rowan, K.M. and Perkins, G.D., 2009. Outcomes following oesophagectomy in patients with oesophageal cancer:
A secondary analysis of the ICNARC case mix Programme database. Critical Care, 13(Suppl 2), p.S1.

(Park et al., 2009)

Pennathur, A., Gibson, M.K., Jobe, B.A. and Luketich, J.D., $2016 . \quad$ Oesophageal carcinoma381(9864), pp.400-412. Available at: $<$ http://www.sciencedirect.com/science/article/pii/S01 40673612606436> [Accessed 17 September 2016].

(Pennathur et al., 2016)

Peters, C.J. and Fitzgerald, R.C., 2007. Systematic Review: The Application of Molecular Pathogenesis to Prevention and Treatment of Oesophageal Adenocarcinoma. [Aliment Pharmacol Ther.] Available at:

$<\mathrm{http}: / /$ www.medscape.com/viewarticle/557839>

[Accessed 15 September 2016].

(Peters and Fitzgerald, 2007)

Relevo, R., 2012. Effective search strategies for systematic reviews of medical tests. [Rockville] NCBI Bookshelf. Available at: $<$ http://www.ncbi.nlm.nih.gov/books/NBK98242/> [Accessed 18 September 2016].

(Relevo, 2012)

Savides, T.J., 2016. Endoscopic Ultrasound. [GastroHep.com] Wiley-Blackwell. Available at: $<$ http://www.gastrohep.com/ebooks/thumbnails.asp?b ook $=1405120819 \& \mathrm{id}=3>$ [Accessed 15 September 2016].

(Savides, 2016)

Schardt, C., Adams, M.B., Owens, T., Keitz, S. and Fontelo, P., 2007. Utilization of the PICO framework to improve searching PubMed for clinical questions. BMC Medical Informatics and Decision Making, 7(1), p.16.

(Schardt et al., 2007)

Scheepers, J.J.G., Cuesta, M.A., Donald L van der Peet and Alexander A F A Veenhof, 2007. Thoracoscopic resection for esophageal cancer: A review of literature3(4). Available at: 
$<$ http://www.ncbi.nlm.nih.gov/pmc/articles/PMC2749

198/> [Accessed 16 September 2016].

(Scheepers et al., 2007)

Schoonjans, F., 2016. MedCalc $\square$ statistics for biomedical research software manual.. Available at: $<$ https://www.medcalc.org/download/medcalcmanual. pdf $>$ [Accessed 27 September 2016].

(Schoonjans, 2016)

Senthilnathan, P., Rajan, P.S., Vaithiswaran, S, R., P, P., C, P. and PMC, E., 2010. Minimally invasive oesophagectomy for carcinoma oesophagus-approaches and options in a high volume tertiary centre.Journal of the Indian Medical Association, 108(10), pp.642-644. Available at: $<$ http://europepmc.org/abstract/med/21510545> [Accessed 16 September 2016].

(Senthilnathan et al., 2010)

Sgourakis, G., Gockel, I., Radtke, A., Musholt, T.J., Timm, S., Rink, A., Tsiamis, A., Karaliotas, C. and Lang, H., 2010. Minimally invasive versus open Esophagectomy: Meta-Analysis of outcomes. Digestive Diseases and Sciences, 55(11), pp.30313040 .

(Sgourakis et al., 2010)

Shen, Y., Feng, M., Tan, L., Wang, H., Li, J. and Xi, Y., 2014. Thoracoscopic esophagectomy in prone versus decubitus position: Ergonomic evaluation from a randomized and controlled study. The Annals of thoracic surgery., 98(3), pp.1072-8 Available at: $<$ http://www.ncbi.nlm.nih.gov/pubmed/25038015> [Accessed 14 September 2016].

(Shen et al., 2014)

Shen, Y., Zhong, M., Wu, W., Wang, H., Feng, M. and Tan, L., 2013. The impact of tidal volume on pulmonary complications following minimally invasive esophagectomy: A randomized and controlled study. The Journal of thoracic and cardiovascular surgery., 146(5), pp.1267-73 Available $<$ http://www.ncbi.nlm.nih.gov/pubmed/23993028> [Accessed 14 September 2016].
(Shen et al., 2013)

Smithers, B.M., Gotley, D.C., Martin, I. and Thomas, J.M., 2007. Comparison of the outcomes between open and minimally invasive Esophagectomy245(2) Available at: $<$ http://www.ncbi.nlm.nih.gov/pmc/articles/PMC1876 975/> [Accessed 15 September 2016].

(Smithers et al., 2007)

StatsDirect Limited, 2000. Heterogeneity in Metaanalysis $(Q, \quad i$-square $) . \quad$ Available at: $<$ http://www.statsdirect.com/help/meta_analysis/heter ogeneity.htm $>$ [Accessed 27 September 2016].

(StatsDirect Limited, 2000)

Taylor, C., 2016. Range rule for standard deviation how to estimate the standard deviation. About.com. Available at: $<$ http://statistics.about.com/od/DescriptiveStatistics/a/Range-Rule-For-Standard-Deviation.htm> [Accessed 27 September 2016].

(Taylor, 2016)

Titcomb, D.R., Burdall, O.C., Fullick, J., P Boddy, A., Blazeby, J., Krysztopik, R., Streets, C., Hollowood, A. and Barham, C.P., 2016. Improved survival following minimally invasive Oesophagectomy compared to open surgery - SAGES abstract archives. SAGES. Available at:

$<$ http://www.sages.org/meetings/annual-

meeting/abstracts-archive/improved-survivalfollowing-minimally-invasive-oesophagectomycompared-to-open-surgery/> [Accessed 15 September 2016].

(Titcomb et al., 2016)

Verghese, R., EJ, H., J, B., R, V.H. and PMC, E., 2009. Minimally invasive surgery compared to open procedures in esophagectomy for cancer: A systematic review of the literature. Minerva Chirurgica, 64(2), pp.135-146. Available at: $<$ http://europepmc.org/abstract/med/19365314>

[Accessed 16 September 2016].(Verghese et al., 2009)

WCRFI, 2015.

Cancer facts \& figures - Worldwide data. World cancer research fund international. Available at: $<$ http://www.wcrf.org/int/cancer-facts- 
International Journal of Trend in Scientific Research and Development (IJTSRD) ISSN: 2456-6470

figures/worldwide-data $>$ [Accessed 15 September 2016].(WCRFI, 2015)

Yamamoto, M., Weber, J.M., Karl, R.C. and Meredith, K.L., 2013. Minimally invasive surgery for Esophageal cancer: Review of the literature and institutional experience20(2) Available at: $<$ https://moffitt.org/File\%20Library/Main\%20Nav/Re search\%20and\%20Clinical\%20Trials/Cancer\%20Con trol\%20Journal/v20n2/130.pdfs [Accessed 15 September 2016]. (Yamamoto et al., 2013)

\section{APPENDICES}

\begin{tabular}{|l|l|l|l|}
\hline Rank & Cancer & $\begin{array}{l}\text { New cases } \\
\text { diagnosed in 2012 } \\
(1,000 \text { s) }\end{array}$ & $\begin{array}{l}\text { Percent of all cancers } \\
\text { (excl. Nonmelanoma } \\
\text { skin cancers) }\end{array}$ \\
\hline $\mathbf{1}$ & Lung & 1,825 & 13.0 \\
\hline $\mathbf{2}$ & Breast & 1,677 & 11.9 \\
\hline $\mathbf{3}$ & Colorectum & 1,361 & 9.7 \\
\hline $\mathbf{4}$ & Prostate & 1,112 & 7.9 \\
\hline $\mathbf{5}$ & Stomach & 952 & 6.8 \\
\hline $\mathbf{6}$ & Liver & 782 & 5.6 \\
\hline $\mathbf{7}$ & Cervix uteri & 528 & 3.7 \\
\hline $\mathbf{8}$ & Oesophagus & 456 & 3.2 \\
\hline $\mathbf{9}$ & Bladder & 430 & 3.1 \\
\hline $\mathbf{1 0}$ & Non-Hodgkin lymphoma & 386 & 2.7 \\
\hline $\mathbf{1 1}$ & Leukaemia & 352 & 2.5 \\
\hline $\mathbf{1 2}$ & Pancreas & 338 & 2.4 \\
\hline $\mathbf{1 3}$ & Kidney & 338 & 2.4 \\
\hline $\mathbf{1 4}$ & Uterus (endometrium) & 320 & 2.3 \\
\hline $\mathbf{1 5}$ & Lip, oral cavity & 300 & 2.1 \\
\hline $\mathbf{1 6}$ & Thyroid & 298 & 2.1 \\
\hline $\mathbf{1 7}$ & Brain, nervous system & 256 & 1.8 \\
\hline $\mathbf{1 8}$ & Ovary & 239 & 1.7 \\
\hline $\mathbf{1 9}$ & Melanoma of skin & 232 & 1.6 \\
\hline $\mathbf{2 0}$ & Gallbladder & 178 & 1.3 \\
\hline $\mathbf{2 1}$ & Larynx & 157 & 1.1 \\
\hline $\mathbf{2 2}$ & Other pharynx & 142 & 1.0 \\
\hline $\mathbf{2 3}$ & Multiple myeloma & 114 & 0.8 \\
\hline $\mathbf{2 4}$ & Nasopharynx & 87 & 0.6 \\
\hline $\mathbf{2 5}$ & Hodgkin lymphoma & 66 & 0.5 \\
\hline $\mathbf{2 6}$ & Testis & 55 & 0.3 \\
\hline $\mathbf{2 7}$ & Kaposi sarcoma & 44 & \\
\hline & & & \\
\hline
\end{tabular}




\section{APPENDIX 1}

Ferlay J, Soerjomataram I, Ervik M, Dikshit R, Eser S, Mathers C, Rebelo M, Parkin DM, Forman D, Bray, F. GLOBOCAN 2012 v1.1, Cancer Incidence and Mortality Worldwide: IARC CancerBase No. 11 [Internet]. Lyon, France: International Agency for Research on Cancer; 2014.

Available from: http:// globocan.iarc.fr, accessed on 16/09/2016.

\section{APPENDIX 2}

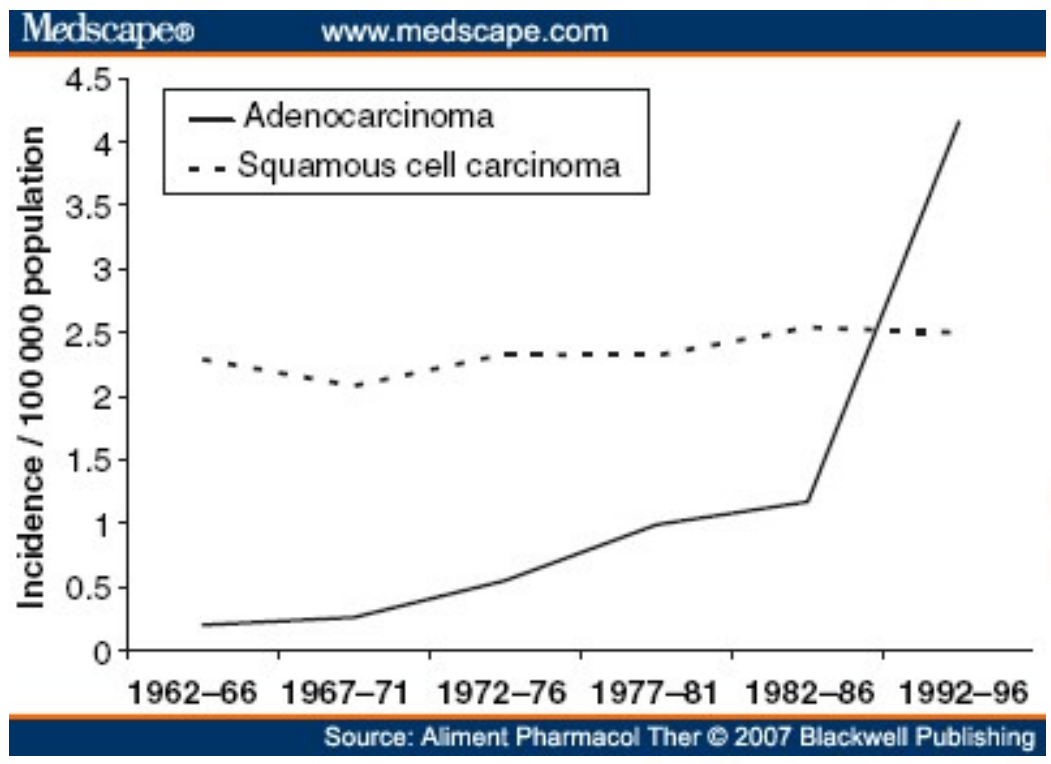

Historical change in the male incidence of oesophageal adenocarcinoma and squamous cell carcinoma (UK data)

Available at: http://onlinelibrary.wiley.com/doi/10.1111/j.1365-2036.2007.03325.x/full

Accessed on 16/5/2016 
International Journal of Trend in Scientific Research and Development (IJTSRD) ISSN: 2456-6470

\section{PPENDIX 3 ---- TNM staging of oesophageal cancer ---}

\begin{tabular}{|c|c|c|c|}
\hline \multicolumn{4}{|c|}{ Primary tumor $(\mathrm{T})$} \\
\hline \multicolumn{4}{|c|}{$\mathrm{T} 1=$ mucosal/submucosal involvement } \\
\hline \multicolumn{4}{|c|}{ T2 = into, but not through, the muscularis propria } \\
\hline \multicolumn{4}{|c|}{$\mathrm{T} 3=$ through the entire wall and into the peri-luminal fat or through the serosa } \\
\hline \multicolumn{4}{|c|}{ T4 = into adjacent organs (aorta, pleura, trachea, pericardium) } \\
\hline \multicolumn{4}{|c|}{ Regional lymph nodes $(\mathrm{N})$} \\
\hline \multicolumn{4}{|c|}{ N0 $=$ no metastatic nodes } \\
\hline \multicolumn{4}{|c|}{ N1 = regional metastatic nodes } \\
\hline \multicolumn{4}{|c|}{ Distant metastasis (M) } \\
\hline \multicolumn{4}{|c|}{ Tumors of the upper thoracic esophagus } \\
\hline \multicolumn{4}{|c|}{ M1a Metastasis in cervical nodes } \\
\hline \multicolumn{4}{|c|}{ M1b Other distant metastasis } \\
\hline \multicolumn{4}{|c|}{ Tumors of the mid thoracic esophagus } \\
\hline \multicolumn{4}{|c|}{ M1a Not applicable } \\
\hline \multicolumn{4}{|c|}{ M1b Non-regional lymph nodes and/or other distant metastasis } \\
\hline \multicolumn{4}{|c|}{ Tumors of the lower thoracic esophagus } \\
\hline \multicolumn{4}{|c|}{ M1a Metastasis in the celiac lymph nodes } \\
\hline \multicolumn{4}{|c|}{ M1b Other distant metastasis } \\
\hline \multicolumn{4}{|c|}{ Tumors of the gastroesophageal junction } \\
\hline \multicolumn{4}{|c|}{ M1 Distant metastasis (not celiac or gastrohepatic ligament notes) } \\
\hline \multicolumn{4}{|c|}{ Esophageal cancer stage groupings [1] } \\
\hline Stage & T-stage & $N$-stage & M-stage \\
\hline 1 & Tis & No & M0 \\
\hline IIA & T2-3 & No & M0 \\
\hline III & T1-2 & N1 & M0 \\
\hline \multirow[t]{2}{*}{ III } & T3 & N1 & Mo \\
\hline & T4 & Any $N$ & M0 \\
\hline IV & Any $T$ & Any $N$ & M1 \\
\hline IVA & Any $T$ & Any $N$ & M1a \\
\hline IVB & Any $T$ & Any $\mathrm{N}$ & M1b \\
\hline
\end{tabular}

Tis, tumor in situ.

Available at - http://www.gastrohep.com/ebooks/thumbnails.asp?book=1405120819\&id=3 


\section{Appendix 4}

Table 1: MIE outcomes in institutional series, case-control studies, and systematic reviews.

\begin{tabular}{|c|c|c|c|c|c|c|}
\hline Study & Type & Leak & $\begin{array}{l}\text { Pn } \\
\text { eu } \\
\text { mo } \\
\text { nia }\end{array}$ & $\begin{array}{l}\mathrm{RL} \\
\mathrm{N} \\
\text { inju } \\
\text { ry }\end{array}$ & $\begin{array}{l}\text { M } \\
\text { orb } \\
\text { idit } \\
y\end{array}$ & Mortality \\
\hline
\end{tabular}

Institutional series

\begin{tabular}{|c|c|c|c|c|c|c|c|}
\hline Luketich et al. [14] & 206 & MIE & $\begin{array}{l}11.7 \\
\%\end{array}$ & $\begin{array}{l}7.7 \\
\%\end{array}$ & $\begin{array}{l}3.6 \\
\%\end{array}$ & - & $1.4 \%$ \\
\hline Bizekis et al. [15] & 50 & MIE & $6 \%$ & - & - & - & $6 \%$ \\
\hline Rajan et al. [16] & 463 & MIE & - & - & - & $\begin{array}{l}16 \\
\%\end{array}$ & $0.9 \%$ \\
\hline Nguyen et al. [17] & 104 & MIE & $9.6 \%$ & 一 & - & $\begin{array}{l}12 . \\
5 \%\end{array}$ & $2.9 \%$ \\
\hline $\begin{array}{l}\text { Ben-David et al. } \\
{[\underline{18}]}\end{array}$ & 105 & MIE & $4 \%$ & $9 \%$ & $7 \%$ & - & $1 \%$ \\
\hline $\begin{array}{l}\text { Ben-David et al. } \\
{[\underline{19}]}\end{array}$ & 18 & MIE & $5.6 \%$ & $\begin{array}{l}16 . \\
7 \%\end{array}$ & - & - & $5.6 \%$ \\
\hline
\end{tabular}

Systematic reviews

or meta-analyses

\begin{tabular}{|c|c|c|c|c|c|c|}
\hline $\begin{array}{l}\text { Gemmill and } \\
\text { McCulloch [20] }\end{array}$ & 1398 & MIE & $7.7 \%$ & $\begin{array}{l}13 . \\
2 \%\end{array}$ & $\begin{array}{l}46 . \\
2 \%\end{array}$ & $2.3 \%$ \\
\hline Verhage et al. [21] & - & Open & & $\begin{array}{l}22 . \\
9 \%\end{array}$ & $\begin{array}{l}60 . \\
4 \%\end{array}$ & $3.8 \%$ \\
\hline $\begin{array}{l}(10 \text { case-control } \\
\text { studies })\end{array}$ & - & MIE & & $\begin{array}{l}15 . \\
1 \%\end{array}$ & $\begin{array}{l}43 . \\
8 \%\end{array}$ & $1.3 \%$ \\
\hline Nagpal et al. [22] & 612 & Open & $\begin{array}{l}\text { No } \\
\text { differ } \\
\text { ence }\end{array}$ & & & No difference \\
\hline $\begin{array}{l}(12 \quad \text { case-control } \\
\text { studies })\end{array}$ & 672 & MIE & $\begin{array}{l}\text { No } \\
\text { differ } \\
\text { ence }\end{array}$ & & & No difference \\
\hline
\end{tabular}


International Journal of Trend in Scientific Research and Development (IJTSRD) ISSN: 2456-6470
Dantoc et al. [23]
Open
$4.4 \%$
(17 case-control studies)
MIE
$3 \%$
Sgourakis et al. Open
[24]
1008 versus
MIE
Total complications lower with MIE
Biere et al. [25]

$1061 \quad \begin{array}{ll}\text { Open } \\ \text { versus }\end{array}$
Trends favoring MIE, but not significant

(1 randomized controlled trial and 9 case-

control studies)

\begin{tabular}{|c|c|c|c|}
\hline & 6347 & Open & $\begin{array}{l}39 . \\
2 \%\end{array} \quad 4 \%$ \\
\hline$[\underline{20}]$ & 1155 & MIE & $\begin{array}{l}38 \\
\%\end{array}$ \\
\hline
\end{tabular}

MIE: minimally invasive esophagectomy.RLN: recurrent laryngeal nerve.

MIE outcomes in institutional series, case-control studies, systematic reviews ((Kim et al., 2012.) Available at-http://www.ncbi.nlm.nih.gov/pmc/articles/PMC3419416/(Accessed on 19/09/2016)

\section{APPENDIX 5 PRISMA Checklist}

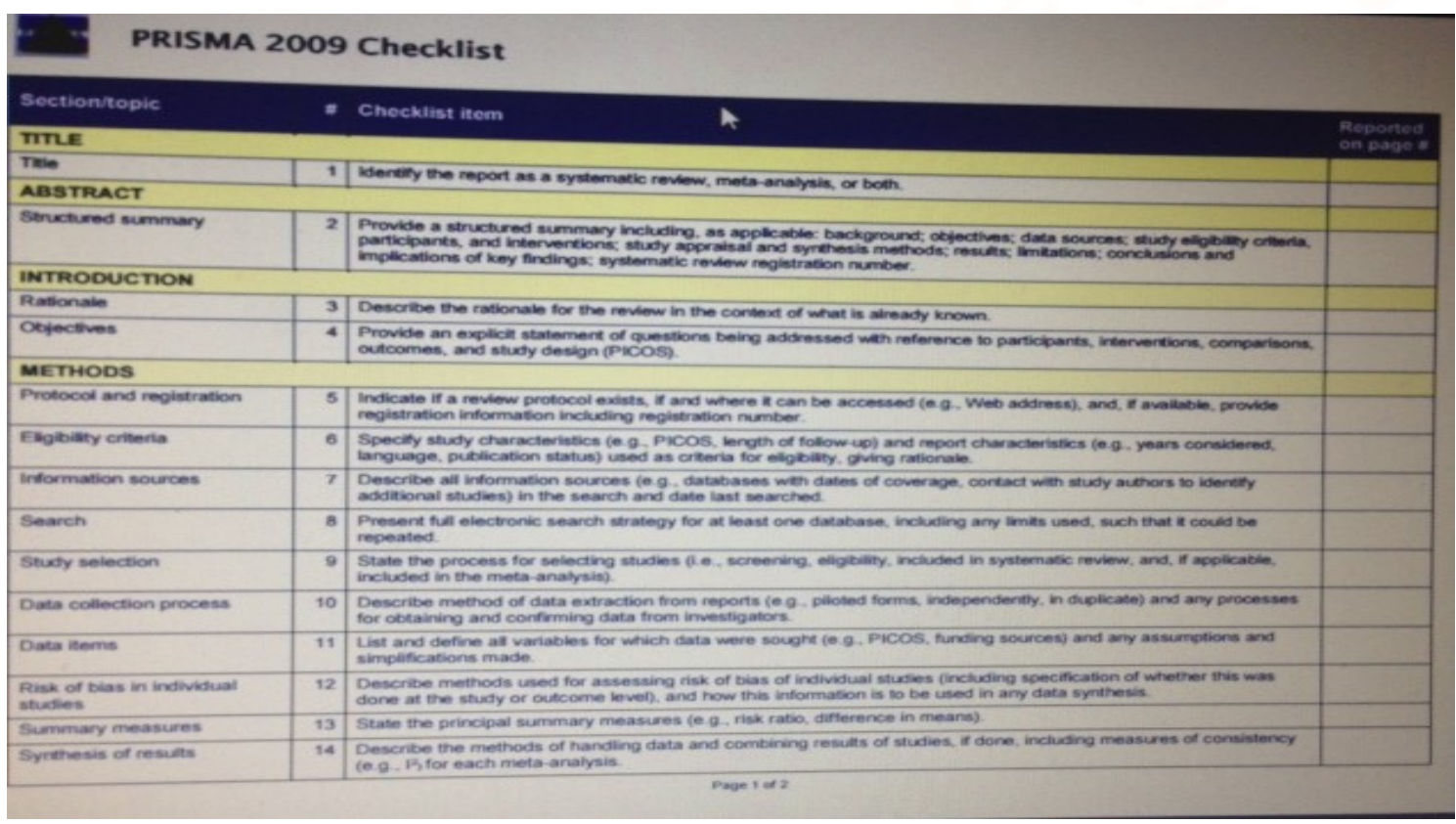


International Journal of Trend in Scientific Research and Development (IJTSRD) ISSN: 2456-6470

Page1 of 2

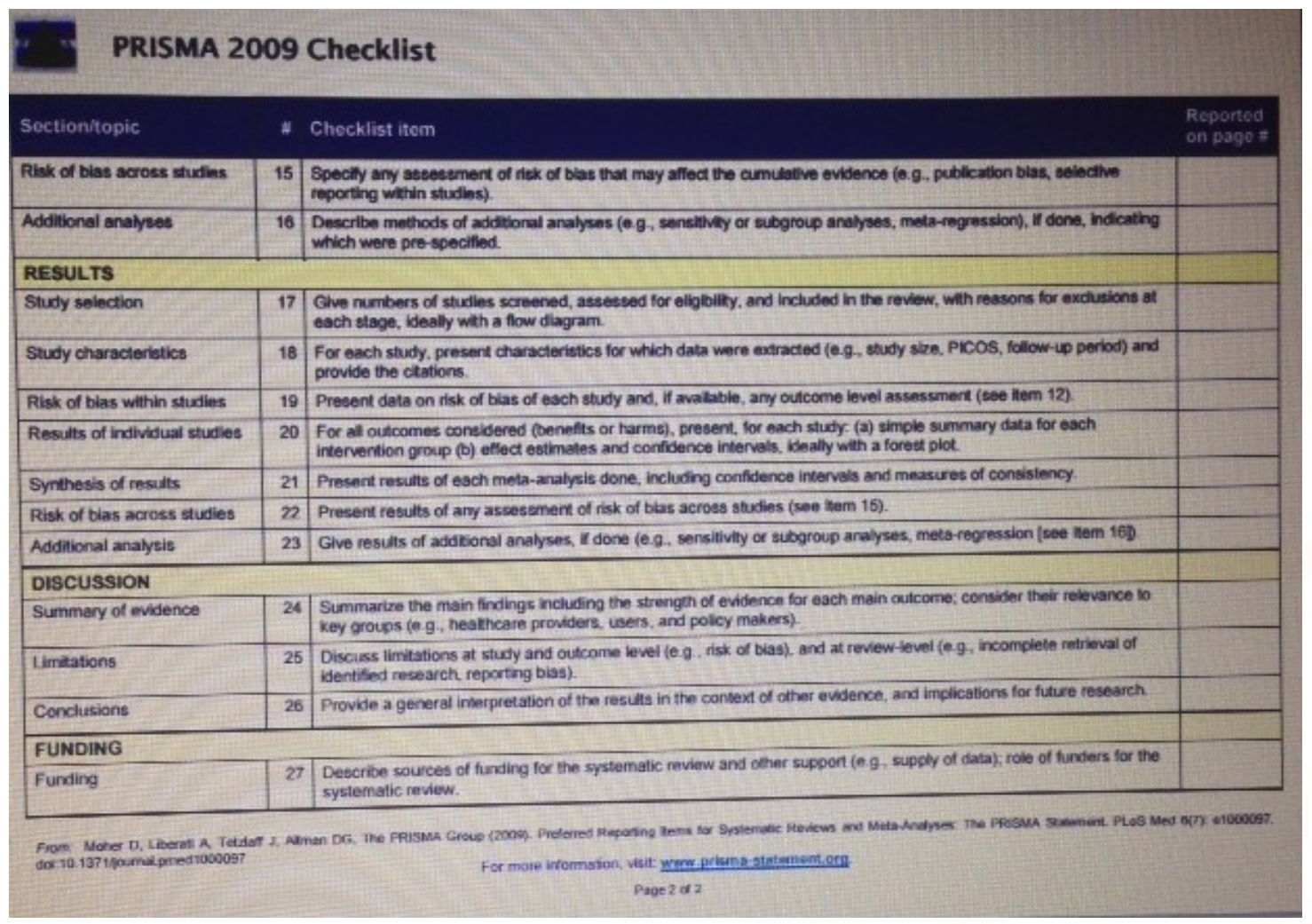

Page 2 of 2

Available online at :http://prisma-statement.org/PRISMAStatement/Checklist.aspx

APPENDIX 6- EXCLUDED STUDIES AFTER FULL TEXT SEARCH
Luketich, J., Pennathur, A., Franchetti, Y., Catalano, P., Swanson, S., Sugarbaker, D.,
Hoyos, D., Maddaus, M., Nguyen, N., Benson, A. and Fernando, H., 2015. Minimally
invasive esophagectomy: Results of a prospective phase II multicenter trial-the eastern
cooperative oncology group (E2202) study. Annals of surgery., 261(4), pp.702-7
Available at: <http://www.ncbi.nlm.nih.gov/pubmed/25575253> [Accessed 14 September
2016].


International Journal of Trend in Scientific Research and Development (IJTSRD) ISSN: 2456-6470

APPENDIX 7

\section{EPHPP- QUALITY APPRAISAL TOOLS}

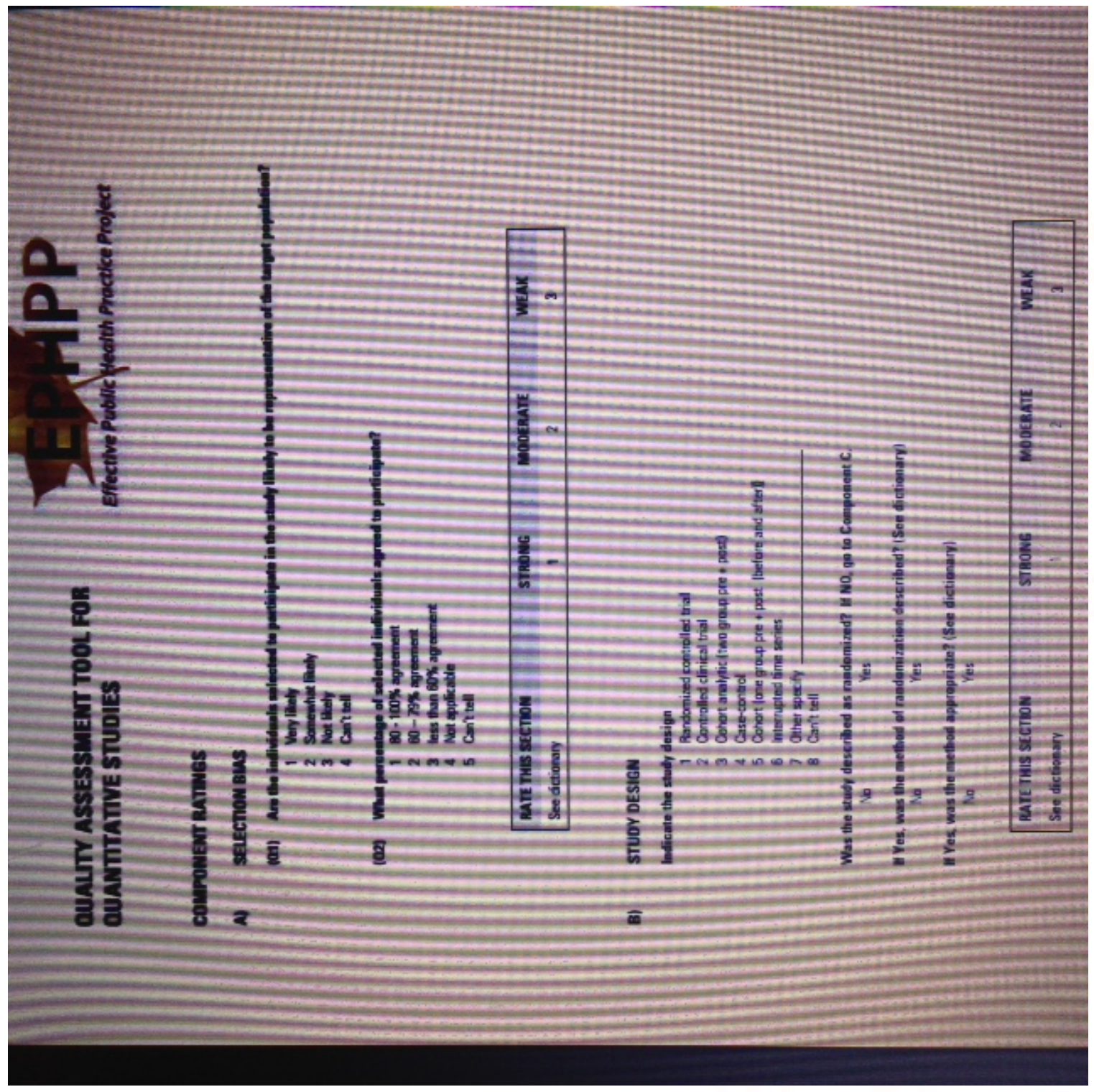

Available at-http://www.ephpp.ca/tools.html

Screen shots taken of the first page of the document

CASP- QUALITY APPRAISAL TOOL 
International Journal of Trend in Scientific Research and Development (IJTSRD) ISSN: 2456-6470

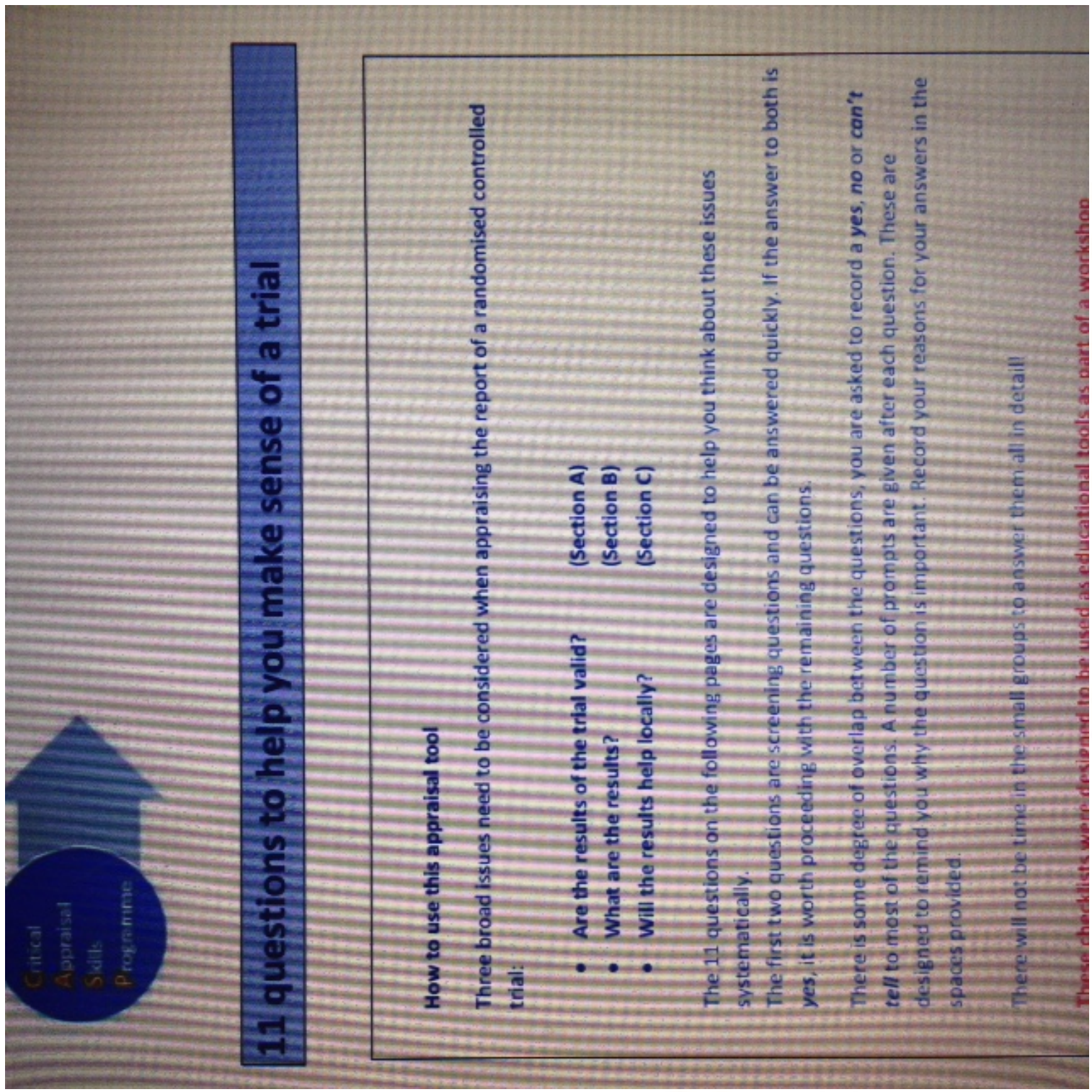

Screen shots taken of the first page of the document Available at http://www.casp-uk.net/ 


\begin{tabular}{|l|l|}
\hline Appendix 8 & Completed search summary for EMBASE via Pubmed \\
\hline
\end{tabular}

1) EMBASE; esophagectomy.ti,ab; 9890 results.

2) EMBASE; Thoracoscopic.ti,ab; 11865 results.

3) EMBASE; laparoscopic.ti,ab; 129079 results.

4) EMBASE; robotic.ti,ab; 27571 results.

5) EMBASE; "minimally invasive".ti,ab; 65598 results.

6) EMBASE; robot-associated.ti,ab; 11 results.

7) EMBASE; "robot-assisted".ti,ab; 8027 results.

8) EMBASE; "minimal access".ti,ab; 2140 results.

9) EMBASE; oesophagectomy.ti,ab; 1783 results.

10) EMBASE; 1 OR 9; 11617 results.

11) EMBASE; 2 OR 3 OR 4 OR 5 OR 6; 207159 results.

12) EMBASE; 10 AND 11; 1980 results.

13) EMBASE; 12 [Limit to: Human and (Languages English) and Publication Year 2000-2016 and (Human Age Groups Adult 18 to 64 years or Aged 65+ years)]; 611 results.

14) EMBASE; 13 [Limit to: Human and (Clinical Trials Randomized Controlled Trial) and (Languages English) and Publication Year 2000-2016 and (Human Age Groups Adult 18 to 64 years or Aged 65+ years)]; 26 results. 